\title{
Acupuncture Methods for Primary Trigeminal Neuralgia: A Systematic Review and Network Meta-Analysis of Randomized Controlled Trials
}

\author{
Zihan Yin $\mathbb{D}^{D}$, Fumin Wang $\mathbb{D}^{D}$, Mingsheng Sun $\mathbb{D}^{D}$, Ling Zhao $\mathbb{D}$, and Fanrong Liang $\mathbb{D}^{-}$ \\ School of Acu-Mox and Tuina, Chengdu University of Traditional Chinese Medicine, Chengdu, China \\ Correspondence should be addressed to Ling Zhao; zhaoling@cdutcm.edu.cn and Fanrong Liang; acuresearch@126.com
}

Received 3 July 2021; Accepted 25 January 2022; Published 21 February 2022

Academic Editor: Rodolfo Parreira

Copyright (c) 2022 Zihan Yin et al. This is an open access article distributed under the Creative Commons Attribution License, which permits unrestricted use, distribution, and reproduction in any medium, provided the original work is properly cited.

\begin{abstract}
Background. Primary trigeminal neuralgia (PTN) is a clinical refractory disorder characterized by excruciating pain that severely impacts the quality of life. Several studies have shown that acupuncture can improve PTN pain. However, the comparative efficacy and safety of acupuncture are unknown. Herein, a systematic review was conducted to compare the efficacy and safety of various acupuncture methods for PTN treatment. Methods. Relevant randomized controlled trials (RCTs) published up to 1 August 2021 were obtained from PubMed, Embase, Cochrane Central Register of Controlled Trials, Web of Science Core Collection, Chinese National Knowledge Infrastructure, Chinese Biomedical Literature Database, CQVIP Database, Wanfang Database, Allied and Alternative Medicine Database, and related registration platforms. Two authors independently selected the studies and obtained data. Cochrane Handbook was used to assess the methodological quality. We put the pain relief as the primary outcome and the response rate and adverse events as the secondary outcomes. Review Manager v5.3, ADDIS v1.16.8, and STATA v15.0 software were used for data analysis. The intraclass correlation coefficient was used to assess the consistency of the two investigators. Results. A total of 58 RCTs with 4,126 participants were obtained. The meta-analysis indicated that five acupuncture methods were superior to conventional medicine (carbamazepine) in pain reduction intensity and response rate. Meanwhile, electronic acupuncture plus manual acupuncture was the most effective therapy since it reduced pain intensity in 11 methods and improved the response rate in 10 interventions. Moreover, six interventions had acceptable adverse events, and none of the included studies reported severe adverse events. However, most pieces of evidence were ranked as critically low. Conclusion. These findings show that acupuncture methods can be effective and safe for PTN. Moreover, electronic acupuncture plus manual acupuncture maybe the best acupuncture treatment for PTN and should be administered to PTN patients. However, additional well-designed and high-quality RCTs should be conducted to verify the above findings in the future. The systematic review is registered with CRD42020221456.
\end{abstract}

\section{Introduction}

Primary trigeminal neuralgia (PTN) is a common neuralgia caused by the compression of an aberrant tissue associated with the trigeminal nerve $[1,2]$. The latest classification system identifies TN as either classical or idiopathic TN based on the degree of neurovascular contact or secondary TN caused by pathology other than neurovascular contact [1]. Meanwhile, it is severe, unilateral, paroxysmal, and recurring pain [3] which can severely impact the quality of life [4]. Al-Quliti and colleagues illustrated that 26.8 people per 100,000 suffer from PTN, usually occurring in middleaged and elderly people [5-7]. PTN is a global public health issue [8]. Several interventions have been used to control PTN, and carbamazepine is the common treatment method since the 1960s [9]. However, many patients do not tolerate pharmacological therapies over a long period due to the carbamazepine side effects $[8,10]$, thus seeking nonpharmacological treatments.

As an ancient nonpharmacological therapy, acupuncture has been used for PTN treatment in China [3, 11-15] for a long period [16]. Numerous studies have also shown that 
acupuncture can be used for PTN treatment [3, 11-17]. Furthermore, the acupuncture analgesic effect is associated with substance $\mathrm{P}(\mathrm{SP})$ and $\beta$-endorphin. Several trials have demonstrated that acupuncture can decrease SP content and enhance the expression of $\beta$-endorphin [18-21]. Although previous systematic reviews [3] have shown that acupuncture methods have good efficacy and safety in improving PTN symptoms, they only focused on a unitary acupuncture method against antiepileptic drugs (carbamazepine)/sham acupuncture (SA). Meanwhile, there are various acupuncture therapies. For instance, the PTN acupuncture methods include manual acupuncture (MA) therapy, acupuncturemoxibustion (AM) therapy, electronic acupuncture (EA) therapy, and fire acupuncture (FA) therapy. Therefore, it is necessary to determine the most appropriate acupuncture methods for PTN therapy.

Network meta-analysis (NMA) analyzes diversified methods from different trials and calculates their relative effects [22-24]. It was applied to compare various interventions simultaneously in a unitary analysis by combining indirect and direct evidence in a network of trials. It may also assist to determine suitable therapeutic methods and illustrate their efficacy and safe application in clinical operations, thus guiding health policies [25]. Thus, this study aimed to compare and rank the efficacy and safety of all known acupuncture therapies on PTN via NMA.

\section{Methods}

The study was registered on PROSPERO (registration number: CRD42020221456) and was conducted following the Preferred Reporting Items for Systematic Review and Meta-Analysis-Network Meta-Analysis (PRISMA-NMA) [26] and the accompanying checklist (Appendix 1).

\subsection{Eligibility Criteria and Exclusion Criteria. The PICOS} (participant, intervention, control, outcome, and study design) criteria were used for inclusion and exclusion analysis.

2.1.1. Types of Studies. All RCT studies reported in English/ Chinese were included, while quasi and cluster RCTs, nonRCTs, case reports, and studies with no detailed data were excluded.

2.1.2. Types of Participants. All PTN patients, regardless of gender, race, and age, were included. Similarly, all types of PTN, including classical TN and idiopathic TN [1] regardless of their etiology, severity, or specific criteria diagnoses, such as diagnostic criteria or references, were included. TN patients without an association with the distinct primary disease were excluded.

2.1.3. Types of Interventions. Herein, only trials using acupuncture as monotherapy or alternative treatments were included. According to Revised Standards for Reporting Interventions in Clinical Trials of Acupuncture
(STRICTA) [27], the expected acupuncture methods included MA, EA, AM, FA, and the like. Moreover, articles describing combinations of these acupuncture methods with conventional medicine (CM) were included. The studies without a clear description of the acupuncture process, such as disinfection and sterilization, acupuncture manipulation, and posttreatment processes, were excluded. Besides, studies using acupoint embedding, acupoint application, acupoint injection, bee venom acupuncture, and other therapies were excluded because of using related drugs. Trials combining acupuncture and herbal medicine, cupping, and blood-letting puncture therapies were also excluded.

2.1.4. Type of Controls. The comparison groups, such as the placebo group (SA), and conventional medicine (carbamazepine, the dosage of carbamazepine should be well documented) were based on different acupuncture methods.

2.1.5. Types of Outcomes. The following studies were included: Those that compared and ranked the efficacy and safety of all acupuncture methods used in PTN. Pain intensity reduction determined using the Visual Analogue Scale (VAS) and Numerical Rating Scale (NRS) was the acceptable primary outcome. The VAS and NRS were converted to the 11 -point NRS ( 0 indicates no pain, and 10 shows the most severe pain) due to their similarity [28]. The response rate and adverse events (AEs) were the secondary outcomes. Reduction of pain intensity and response rate were used to evaluate the efficacy of intervention methods, while $\mathrm{AE}$ was used to assess the safety of intervention methods. Outcome measures that were not relevant to PTN were excluded.

2.2. Search Strategy. Relevant studies were obtained from Embase, PubMed, Cochrane Central Register of Controlled Trials (CENTRAL), Web of Science Core Collection (WOS), and the Chinese database of the Chinese Biomedical Literature Database (CBM), Chinese National Knowledge Infrastructure (CNKI), Chinese Science and Technology Periodical Database (CQVIP), and Wanfang Database (WF) up to 1 August 2021. Furthermore, clinical trial registries (World Health Organization International Clinical Trials Registry Platform (WHO ICTRP), Clinical Trials, and Chinese Clinical Trial Register (ChiCTR)) and Allied and Complementary Medicine Database (AMED) were used as supplements. There were three major search terms: (1) clinical conditions: PTN, classical TN, and idiopathic TN; (2) acupuncture methods: manual acupuncture, electroacupuncture, and acupuncture-moxibustion; and (3) study design: randomized clinical controlled trial. A combination of subject terms and free words was used with "and," "or" to connect the words. The Chinese and English search strategies were similar. The search strategies of each database are shown in Appendix 2. 
2.3. Study Selection and Data Extraction. Two investigators independently selected the studies and extracted data. We first read the study title and abstract to identify duplicate research and then uploaded the remaining part to NoteExpress V.3.0. The two reviewers (ZY and FW) preliminary screened the titles/abstracts to find suitable articles and then downloaded and read the studies. Finally, the 2 researchers each read the residual full-text studies to select those that meet the inclusion criteria. A 3rd party (LZ or FL) resolved any controversy.

A standardized Microsoft Excel 2010 sheet was used to extract data, such as study identity (first author, publication year, and country), study design, diagnostic criteria, characteristics of patients (age, gender, and sample size), details of intervention group and control group, treatment courses, outcomes (primary and secondary outcomes), and results. The corresponding/first author of the article was requested for additional information in ambiguous or insufficient detail cases. Besides, a description was added to the final result when the data details were not available. The selection procedure was outlined following the PRISMA flowchart.

2.4. Study Quality Assessment. Two authors assessed the risk of bias (ROB) in all eligibility trials using the Cochrane Collaboration ROB assessment tool 2.0 [29]. Risk levels of randomization process, deviations from intended interventions, missing outcomes data, measurement of the outcome, and selection of the reported results were indicated as "low," "some concerns," or "high." A 3rd party (LZ or FL) resolved any misunderstanding.

2.5. Statistical Analysis. RevMan 5.3 software was used for data analysis. The 3-arm trials were divided into 2 -arm trials for all possible combinations. A pooled mean difference (MD) for continuous outcomes or relative risk (RR) of dichotomous variable data with $95 \%$ confidence intervals (CI) was presented for each intervention. The random effects model adopted by the DerSimonian-Laired method was used as a conservative estimate [30]. The $I^{2}$ statistic and $p$ values were determined for statistical heterogeneity analysis. $p<0.05$ and $I^{2}>75 \%$ indicated significant heterogeneity. The Bayesian network analysis framework and Markov Chain Monte Carlo (MCMC) method [31] were used to assess and process a priori data via the Aggregate Data Drug Information System (ADDIS V.1.16.8 software, Drugis, Groningen, NL). The parameters were 4 Markov chains for simulation at 50,000 simulation iterations to determine their posterior distributions. Moreover, the first 20,000 simulation iterations were used to eliminate the initial value's impacts and the last 30,000 were used for sampling. The node-splitting method was used to integrate direct and indirect multiple-treatment comparisons of the RCTs [32]. STATA version 15.0 software (StataCorp LP, Texas, USA) was used for network plot analysis, where node sizes indicated the number of study patients and connection sizes showed the number of trials in each treatment. Finally, the node-splitting method was also used to assess the local inconsistency [33]. $p<0.05$ was considered a significant statistical difference between indirect and direct multiple-treatment comparisons. Either the inconsistency model or consistency model was used. The model convergence is the potential scale reduced factor (PSRF), and PSRF close to 1 indicates successful convergence [34].

2.6. Publication Bias. A funnel plot was used to assess reporting bias since over 10 studies were evaluated in the meta-analysis.

2.7. Quality of Evidence. The Grades of Recommendations, Assessment, Development, and Evaluation (GRADE) was used to assess the overall quality of the evidence $[35,36]$ and ranked it as "high," "moderate," "low," and "critically low."

2.8. Assessing Reviewer Agreement. Since the two reviewers independently selected and extracted data and evaluated the quality of RCTs, the intraclass correlation coefficient (ICC) [37] was determined to assess their consistency (ICC score, $0.95)$.

\section{Results}

3.1. Study Selection. A total of 1187 studies were obtained, and only 58 RCTs $[11,17,38-93]$ with 4126 patients were selected for the systematic review. The selection process is presented in Figure 1.

3.2. Study Characteristics. All trials were incorporated in the final Bayesian network meta-analysis. A total of 56 RCTs [11, 38-51, 53-93] were written in Chinese, while two studies $[17,52]$ were published in English. All the 58 articles were reported between 2004 and 2021. The studies were grouped at a 1:1 ratio. The ages of patients were between 38 and 64 years except for six trials, which did not indicate the ages $[71,77,79,88-90]$. The included studies had more women than men with sample sizes between 24 and 217. The treatment duration ranged between 10 days and 90 days (mean, 20-30 days). MA, EA, AM, FA, SA, CM (carbamazepine) treatments, and their combination therapies were used in the included studies. The studies had different acupoints in all acupuncture therapies. However, the Xia Guan (ST 7), He Gu (LI4), and Tai Yang (EX-HN5) were the common acupoints. Moreover, the mean daily usage of carbamazepine was between 0.3 and $0.8 \mathrm{~g}$. The response rate was the frequently used outcome measures in most studies. The characteristics of the included studies are shown in Table 1.

3.3. Study Quality Assessment. The methodology quality of the included RCTs was assessed. (1) Randomisation process: only two trials $[45,58]$ were ranked as "low risk" because they had detailed information, while 56 trials were ranked as "unclear" due to insufficient information. (2) Deviations from intended interventions: all trials had insufficient information on intended interventions and were ranked as "some concerns." (3) Missing outcomes data: all RCTs were ranked as "low risk" due to the complete implementation plan. (4) Measurement of the outcome: a total of 56 trials were ranked as "some concerns" and only two $[17,73]$ as 


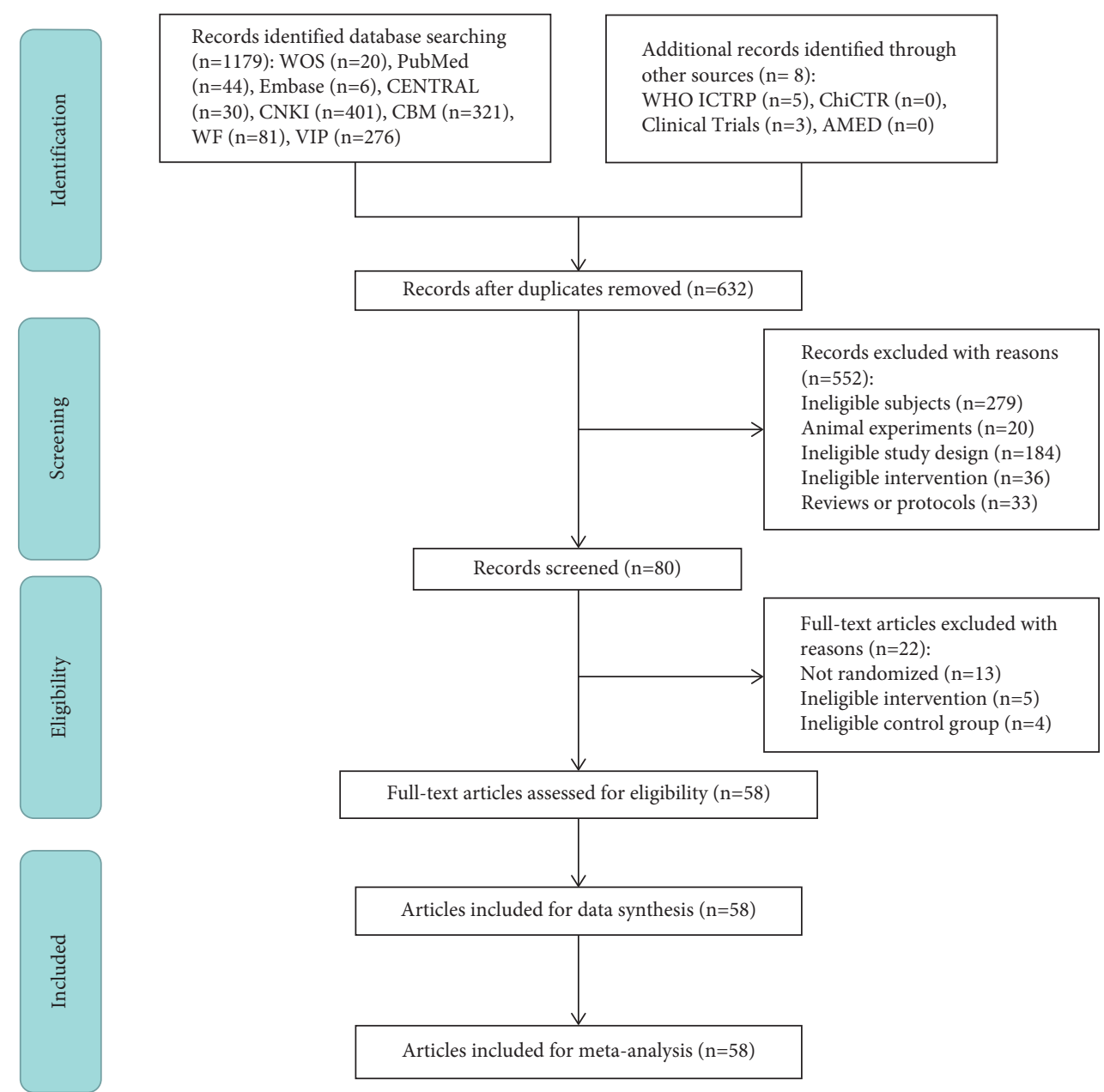

Figure 1: The PRISMA flowchart of selection process.

"low risk." (5) Selection of the reported results: all the RCTs were ranked as "some concerns" due to insufficient information. The quality assessment detail of the RCTs is shown in Figure 2.

\subsection{Pairwise Meta-Analysis Results}

3.4.1. Reduction in Pain Intensity. A total of 14 pairwise meta-analyses were conducted to compare the pain reduction intensity of various therapies (Table 2). MA was highly effective in reducing pain intensity compared to SA (1 RCT, MD, 1.66; 95\% CI: 0.93-2.39) and CM (9 RCTs, MD, 1.14; 95\% CI: $0.48-1.80)$. EA efficacy was statistically different than CM efficacy (1 RCT, MD, 1.07; 95\% CI: 0.09-2.05). Furthermore, MA + EA efficacy was statistically different than MA efficacy (1 RCT, MD, 3.18; 95\% CI: 2.43-3.93) and EA efficacy (2 RCTs, MD, 1.25; 95\% CI: 0.78-1.72). $\mathrm{MA}+\mathrm{CM} \quad(7$ RCTs, $\mathrm{MD}, 1.19 ; 95 \% \mathrm{CI}:$ 0.55-1.84), $\mathrm{AM}+\mathrm{CM} \quad(3 \mathrm{RCTs}, \mathrm{MD}, \quad 1.88 ; 95 \%$ CI: 0.87-2.90), and EA + CM (2 RCTs, MD, 1.22; 95\% CI: $0.42-2.02)$ were highly effective in reducing pain intensity than CM. However, MA + CM efficacy was statistically different than SA + CM efficacy (1 RCT, MD, 1.60; 95\% CI: $0.32-2.88)$. Besides, no statistical difference was found between MA and AM, MA and FA, EA and MA, EA and FA, $\mathrm{AM}$ and $\mathrm{CM}, \mathrm{EA}+\mathrm{CM}$, and $\mathrm{MA}+\mathrm{CM}$.

3.4.2. Response Rate. A total of 13 pairwise meta-analyses were conducted to compare the response rates of different treatments (Table 3). MA efficacy was significantly different than the CM efficacy (25 RCTs, RR, 1.21; 95\% CI: 1.15-1.27) and SA efficacy (1 RCT, RR, 1.68; 95\% CI: 1.17-2.42). $\mathrm{AM}+\mathrm{CM}$ (3 RCTs, RR, 1.25; 95\% CI: 1.10-1.42) and MA + CM (10 RCTs, RR, 1.20; 95\% CI: 1.14-1.26) were highly effective compared with CM. MA + EA was highly effective than MA (1 RCT, RR, 1.50; 95\% CI: 1.13-1.99) and EA (2 RCTs, RR, 1.33; 95\% CI: 1.03-1.72). However, no statistical difference was found between MA and FA, MA and AM, EA and MA, EA/ $\mathrm{AM}$ and $\mathrm{CM}, \mathrm{EA}+\mathrm{CM}$, and $\mathrm{CM} / \mathrm{MA}+\mathrm{CM}$.

\subsection{Network Meta-Analysis Results}

3.5.1. Network Plot of Different Interventions. A total of 11 therapies were used (AM, AM + CM, EA, EA + CM, FA, MA, $\mathrm{MA}+\mathrm{CM}, \mathrm{MA}+\mathrm{EA}, \mathrm{CM}, \mathrm{SA}$, and SA + CM). The network plots of these treatments are shown in Figure 3. A total of 36 trials with 2,308 participants using 11 methods indicated 


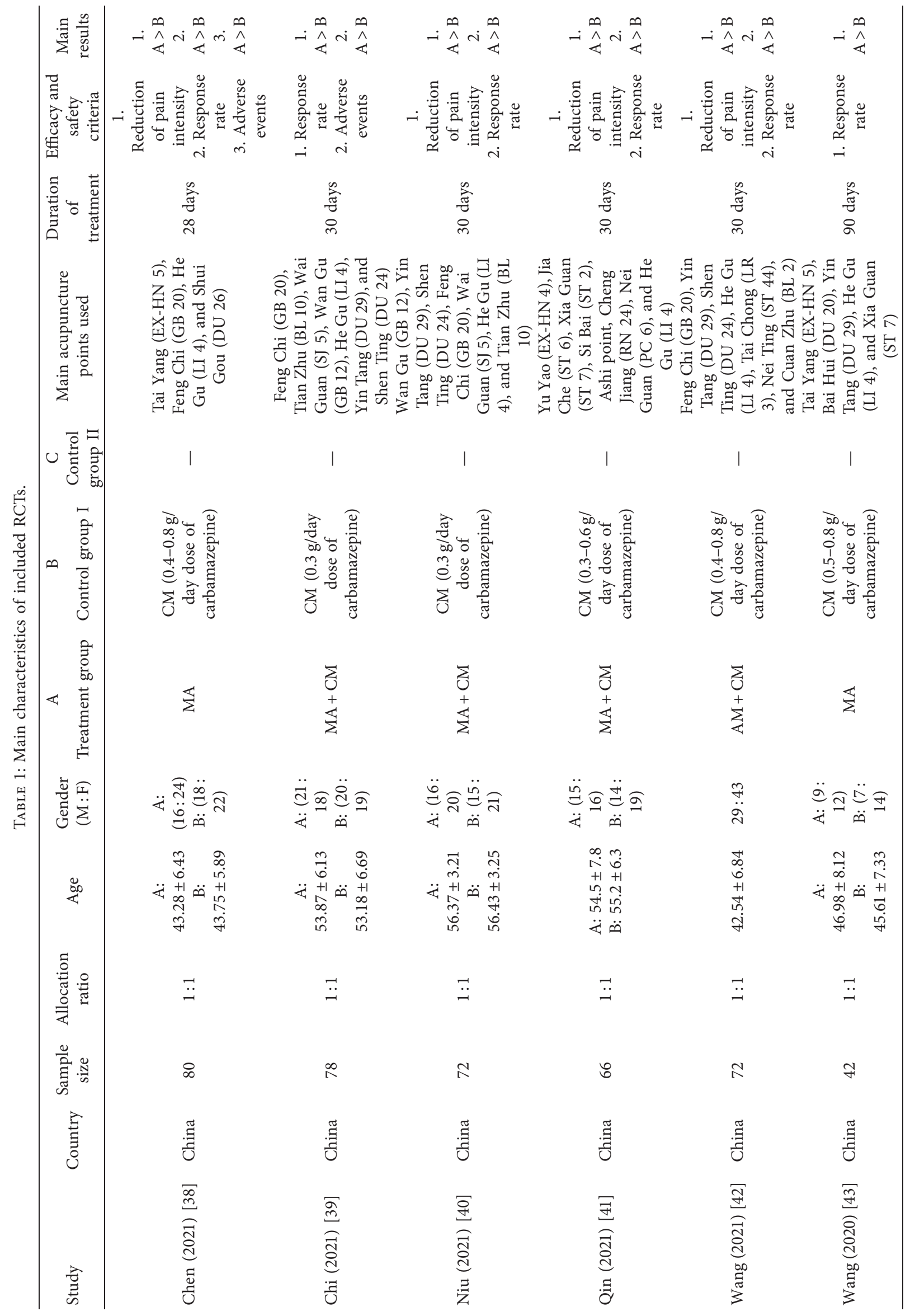




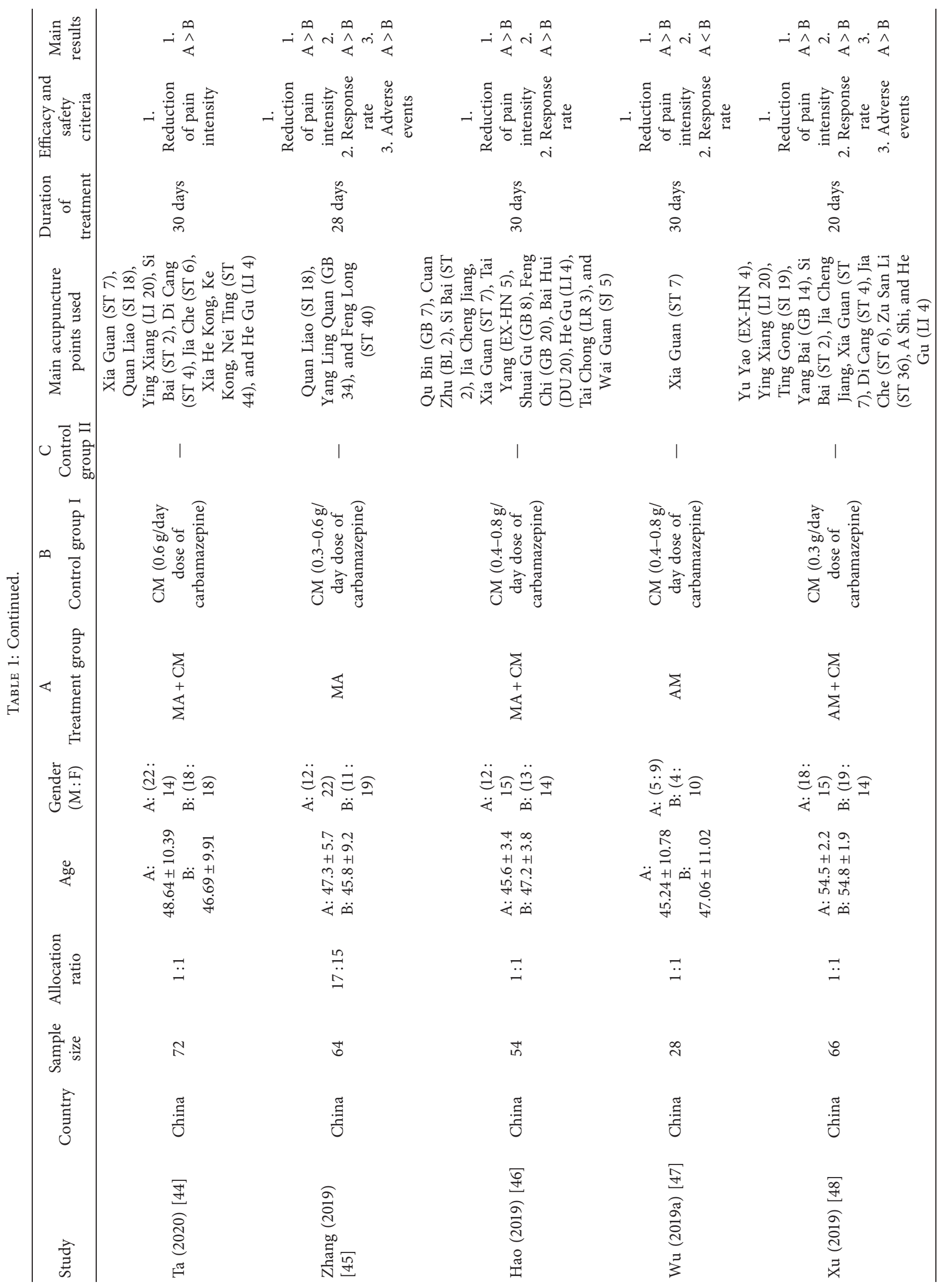




\begin{tabular}{|c|c|c|c|c|c|c|}
\hline 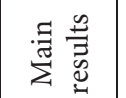 & $-i \hat{\leftrightarrow}$ & $-i \stackrel{\infty}{v} i \stackrel{\infty}{v}$ & 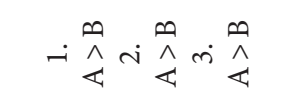 & $-i \stackrel{\infty}{\leftrightarrow}$ & $-i \stackrel{\infty}{\leftrightarrow}$ & 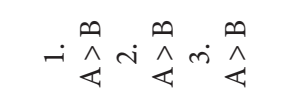 \\
\hline 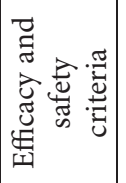 & 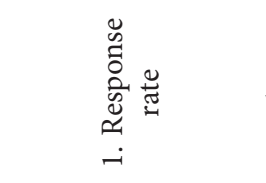 & 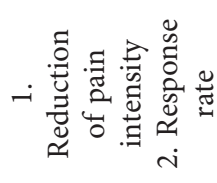 & 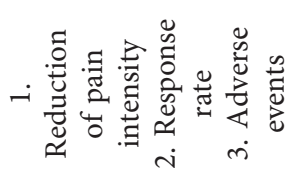 & 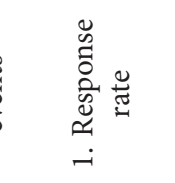 & 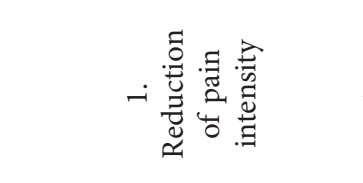 & 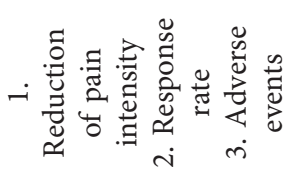 \\
\hline 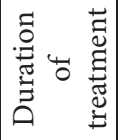 & 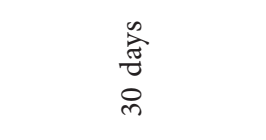 & $\begin{array}{l}\text { } \\
\text { त्र } \\
\dot{m}\end{array}$ & $\begin{array}{l}\text { 密 } \\
0 \\
0\end{array}$ & 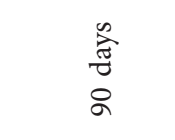 & 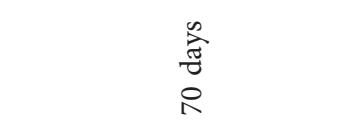 & 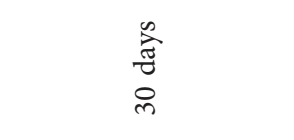 \\
\hline 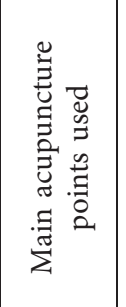 & 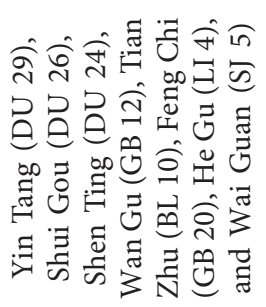 & 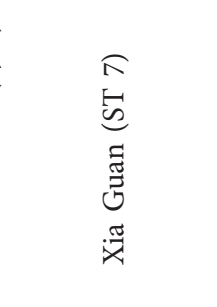 & 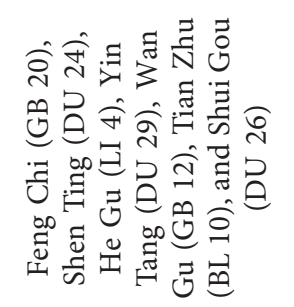 & 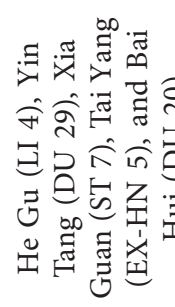 & 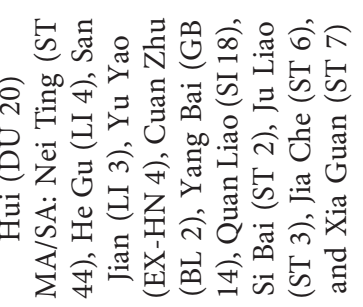 & 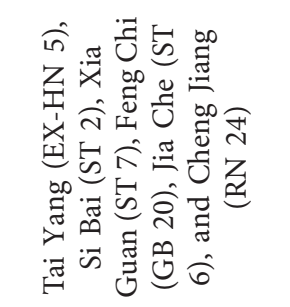 \\
\hline 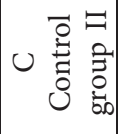 & 1 & 1 & I & I & 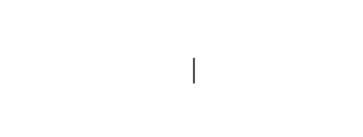 & 1 \\
\hline $\begin{array}{c}\vec{a} \\
\overrightarrow{0} \\
0 \\
\vdots \\
\overrightarrow{0} \\
\overrightarrow{0} \\
\overrightarrow{0} \\
0\end{array}$ & 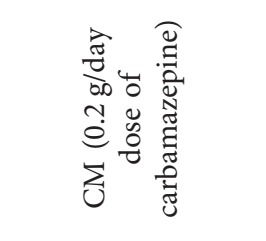 & $\sum_{4}$ & 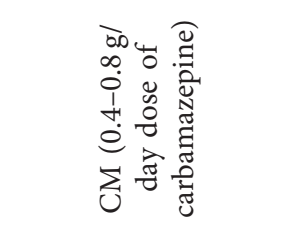 & 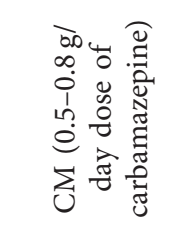 & 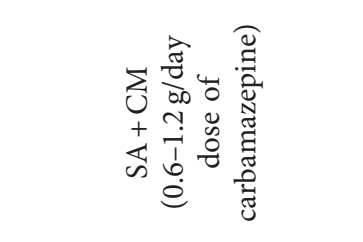 & 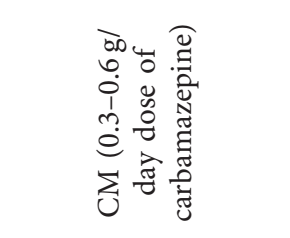 \\
\hline 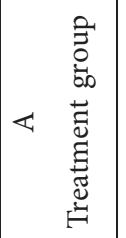 & $\mathbb{\Sigma}$ & $\mathbb{\Sigma}$ & 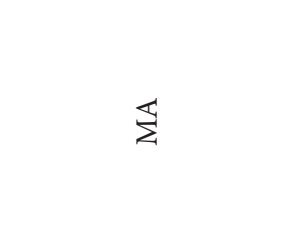 & 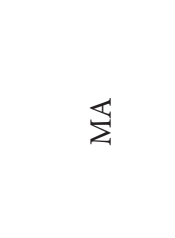 & 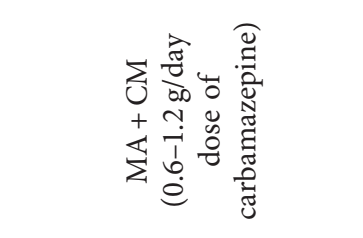 & $\begin{array}{l}\sum_{0} \\
+ \\
+ \\
\text { I. }\end{array}$ \\
\hline 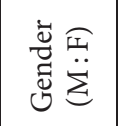 & 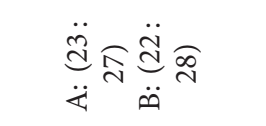 & 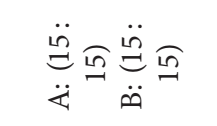 & 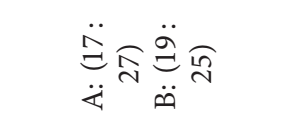 & 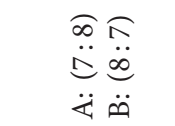 & 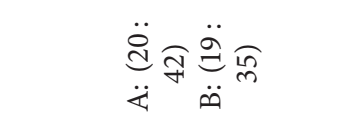 & 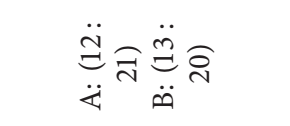 \\
\hline$\stackrel{\infty}{<}$ & 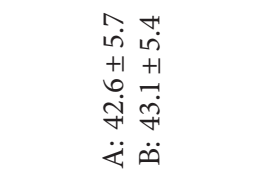 & 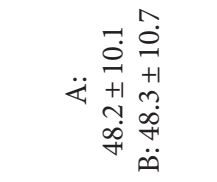 & 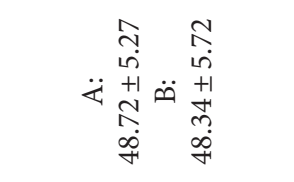 & 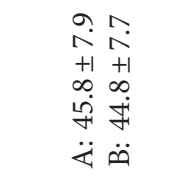 & 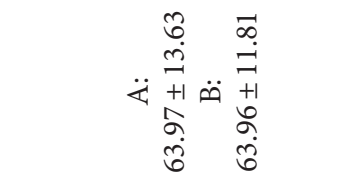 & 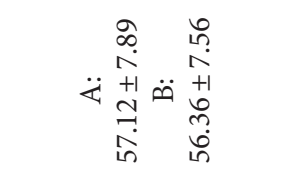 \\
\hline 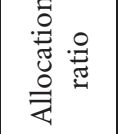 & $\ddot{ت}$ & $\ddot{ت}$ & $\ddot{ت}$ & $\ddot{ت}$ & $\begin{array}{l}\tilde{m} \\
\ddot{m}\end{array}$ & $\ddot{ت}$ \\
\hline 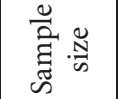 & 8 & 8 & $\infty$ & in & 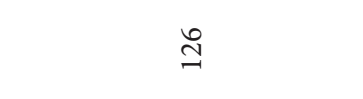 & 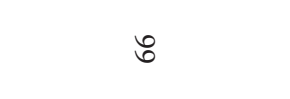 \\
\hline 氧 & ]ี & 肎 & 节 & 节 & : & : \\
\hline$\stackrel{\vec{D}}{\text { s }}$ & 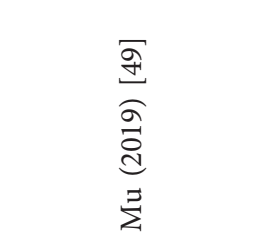 & 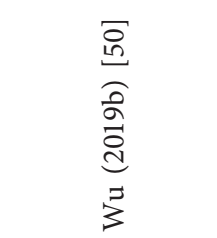 & 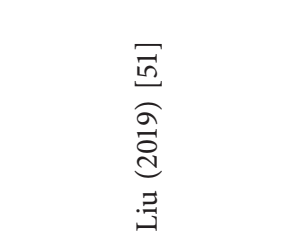 & 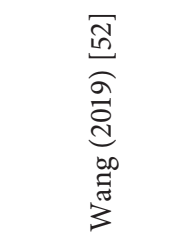 & 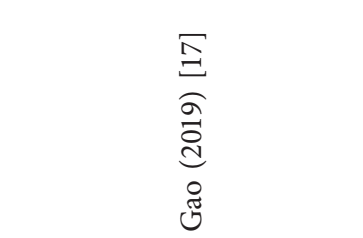 & 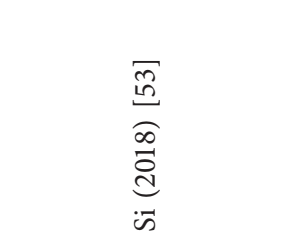 \\
\hline
\end{tabular}




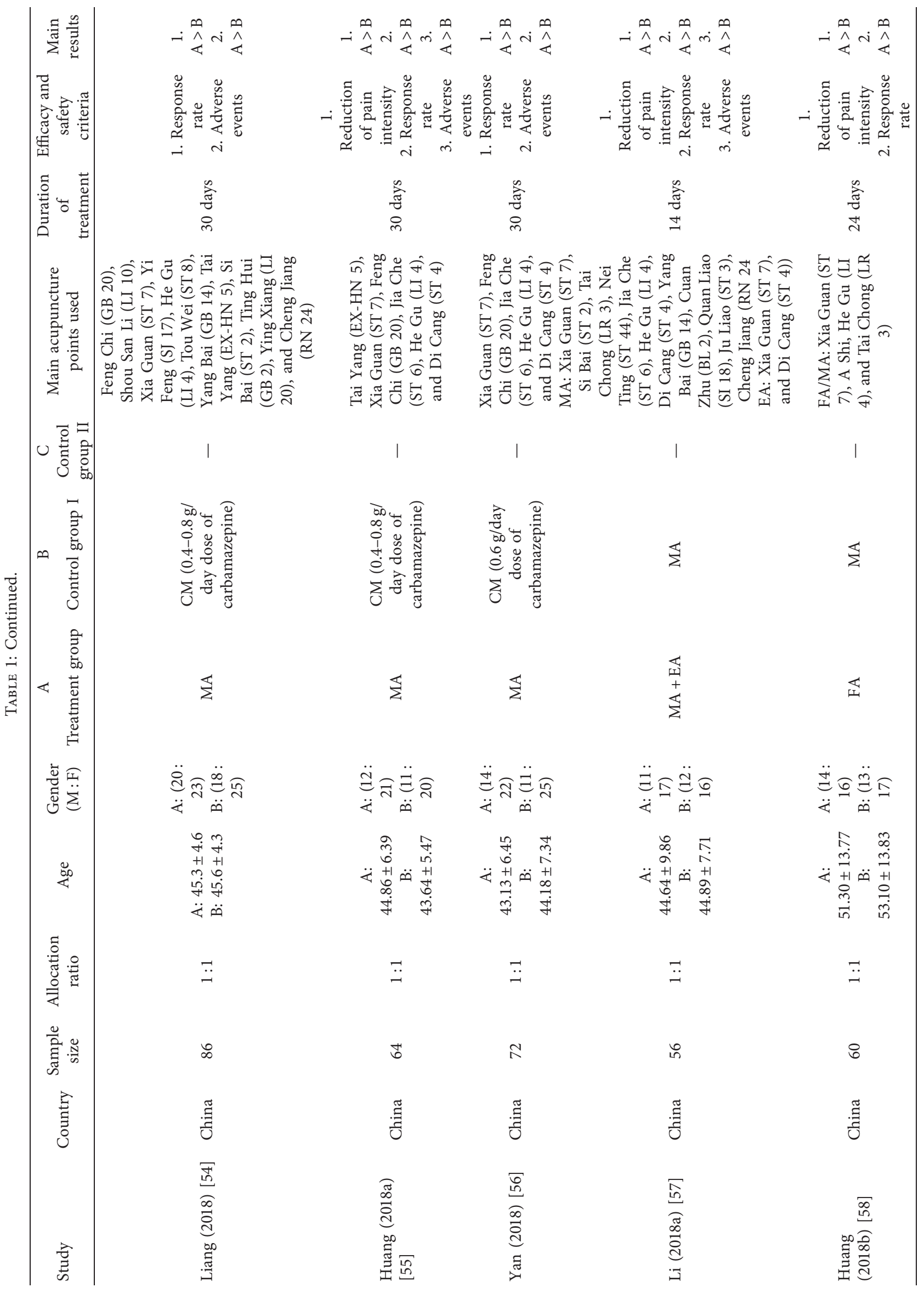




\begin{tabular}{|c|c|c|c|c|c|c|}
\hline 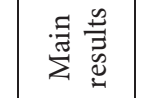 & $-i \stackrel{\wedge}{\wedge} i \stackrel{\infty}{\leftrightarrow}$ & $-i \stackrel{\wedge}{\wedge} i \stackrel{\infty}{\hat{\psi}}$ & $-\dot{\leftrightarrow} \hat{\psi}$ & $-i \stackrel{\infty}{\wedge} i \stackrel{\infty}{\leftrightarrow}$ & $-\dot{n}$ & 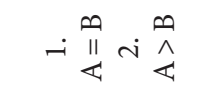 \\
\hline 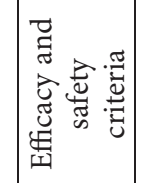 & 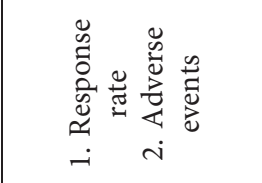 & 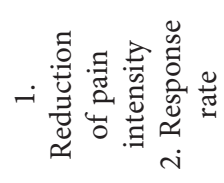 & 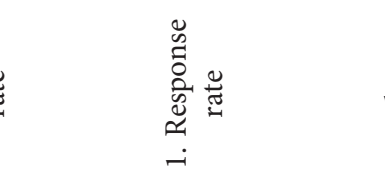 & 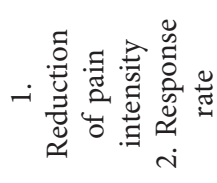 & 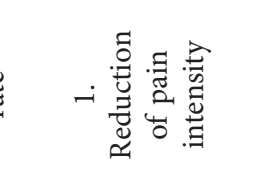 & 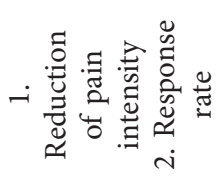 \\
\hline 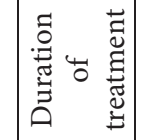 & $\begin{array}{l}\text { co } \\
\text { है } \\
0\end{array}$ & $\begin{array}{l}\text { है } \\
\vec{\tau}\end{array}$ & 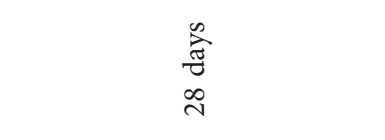 & 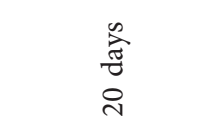 & $\begin{array}{l}\frac{\infty}{\mathrm{E}} \\
\frac{\mathrm{d}}{\sigma}\end{array}$ & $\begin{array}{l}\stackrel{\infty}{\tilde{\sigma}} \\
\underset{c}{\infty} \\
\stackrel{\sim}{N}\end{array}$ \\
\hline 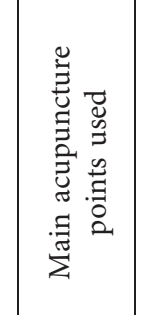 & 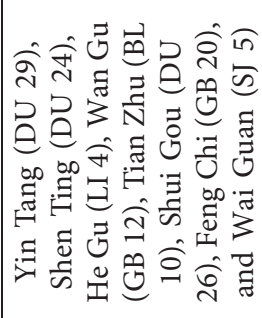 & 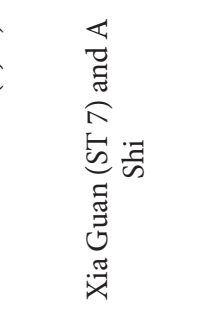 & 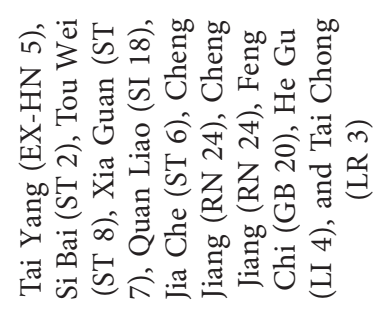 & 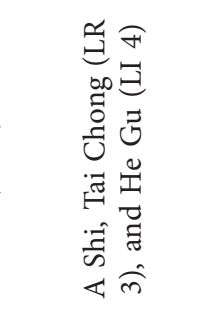 & 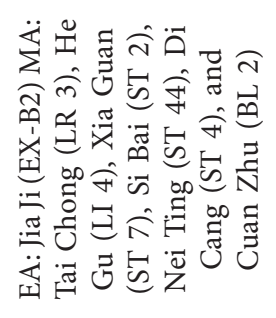 & 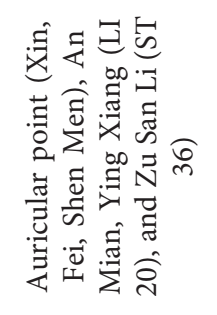 \\
\hline 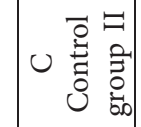 & 1 & 1 & 1 & 1 & I & 1 \\
\hline 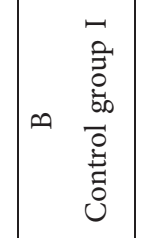 & 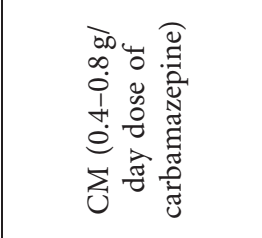 & 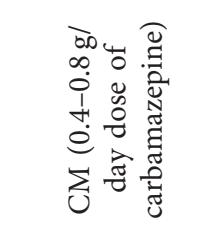 & 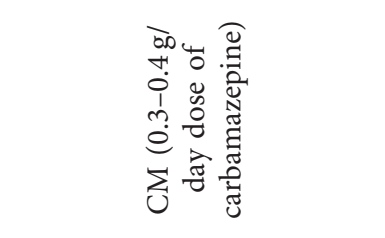 & 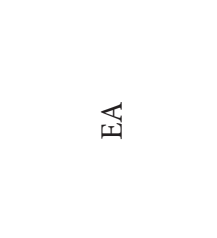 & 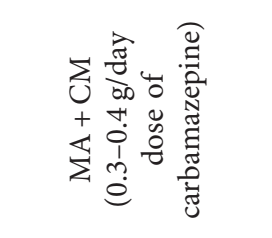 & 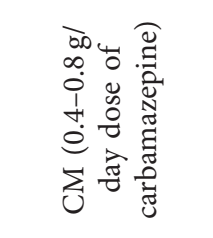 \\
\hline 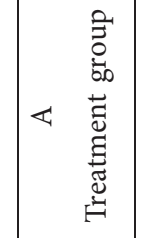 & 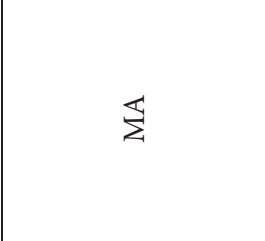 & $\begin{array}{l}\sum_{0} \\
\sum_{i}^{+}\end{array}$ & $\begin{array}{l}\sum_{j} \\
+ \\
\sum_{1}^{+}\end{array}$ & \begin{tabular}{l}
$\mathbb{I}$ \\
+ \\
\multirow{4}{*}{}
\end{tabular} & 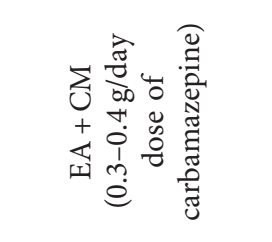 & $\begin{array}{l}\sum_{0} \\
+ \\
\vdots \\
\sum\end{array}$ \\
\hline 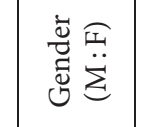 & 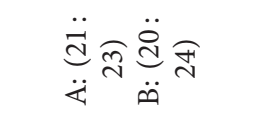 & 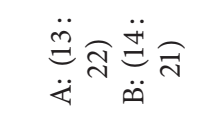 & 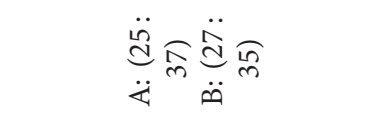 & $\underset{\ddot{\dot{\varphi}}}{\ddot{\infty}} \underset{\stackrel{\sigma}{\sigma}}{\ddot{\theta}}$ & 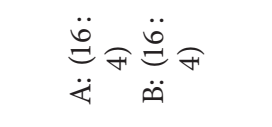 & 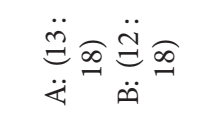 \\
\hline$\stackrel{\circ}{<}$ & 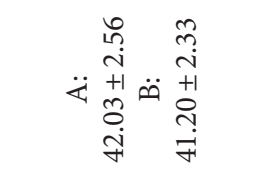 & 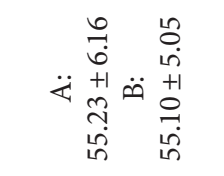 & 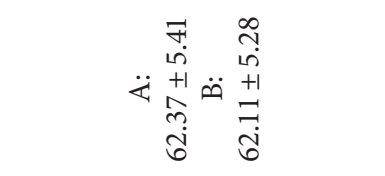 & 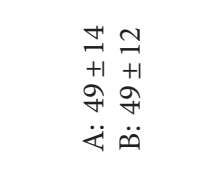 & $\begin{array}{ll} & \\
++ & \\
+1 & +1 \\
0 & 0 \\
\ddot{4} & \ddot{n}\end{array}$ & 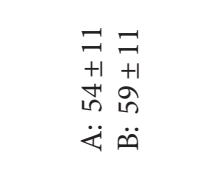 \\
\hline 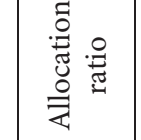 & $\ddot{ت}$ & $\ddot{ت}$ & $\ddot{ت}$ & $\ddot{ت}$ & $\ddot{ت}$ & $\ddot{ت}$ \\
\hline 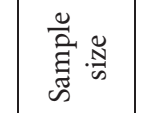 & $\infty$ & $R$ & $\stackrel{\mathbb{J}}{\sim}$ & $\stackrel{i}{\circ}$ & 우 & ช్ \\
\hline 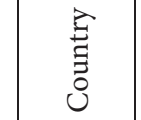 & 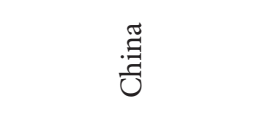 & 苞 & : & 节 & 节 & : \\
\hline$\widehat{\hat{E}}$ & 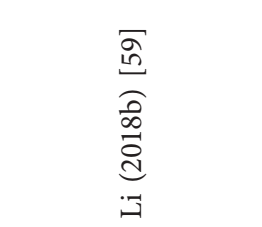 & 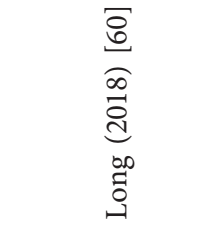 & 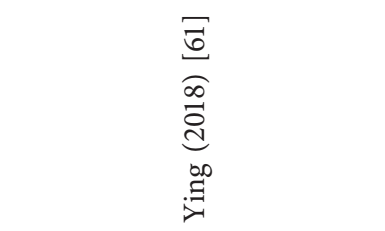 & 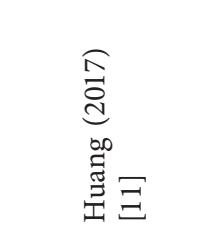 & 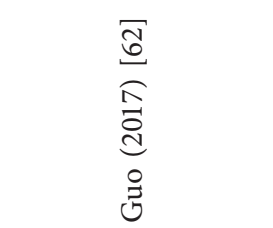 & 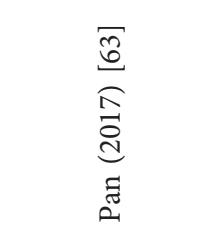 \\
\hline
\end{tabular}




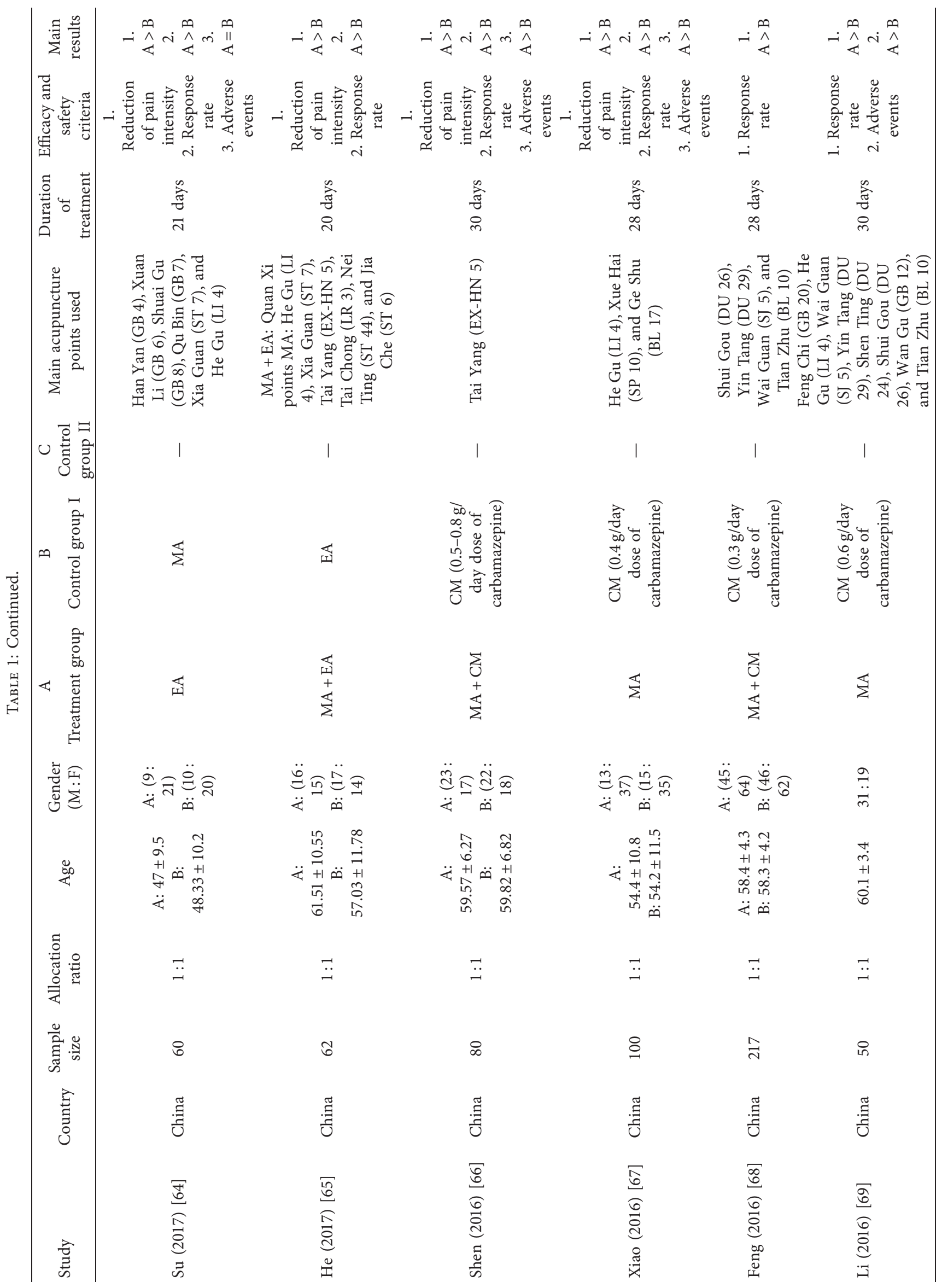




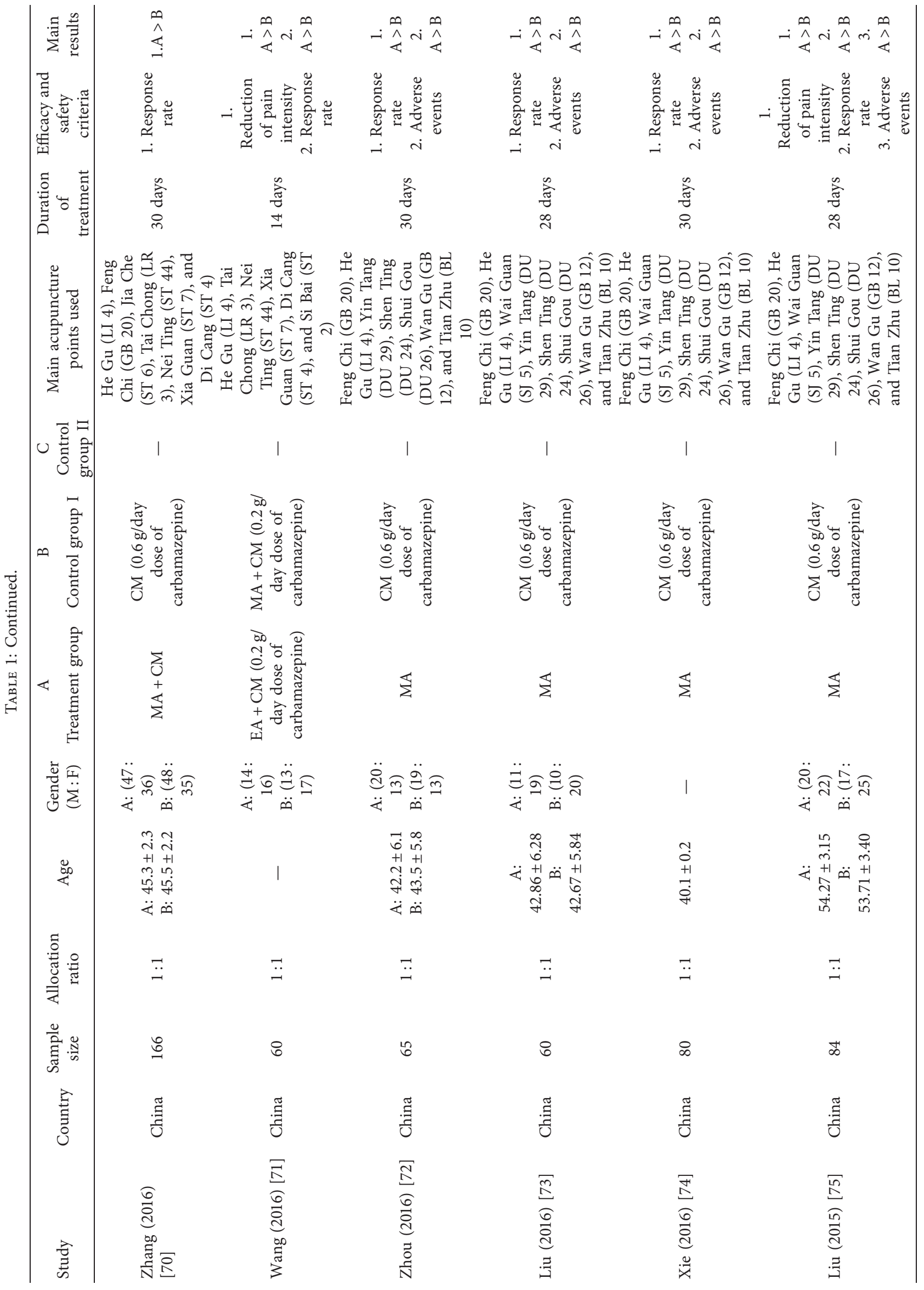




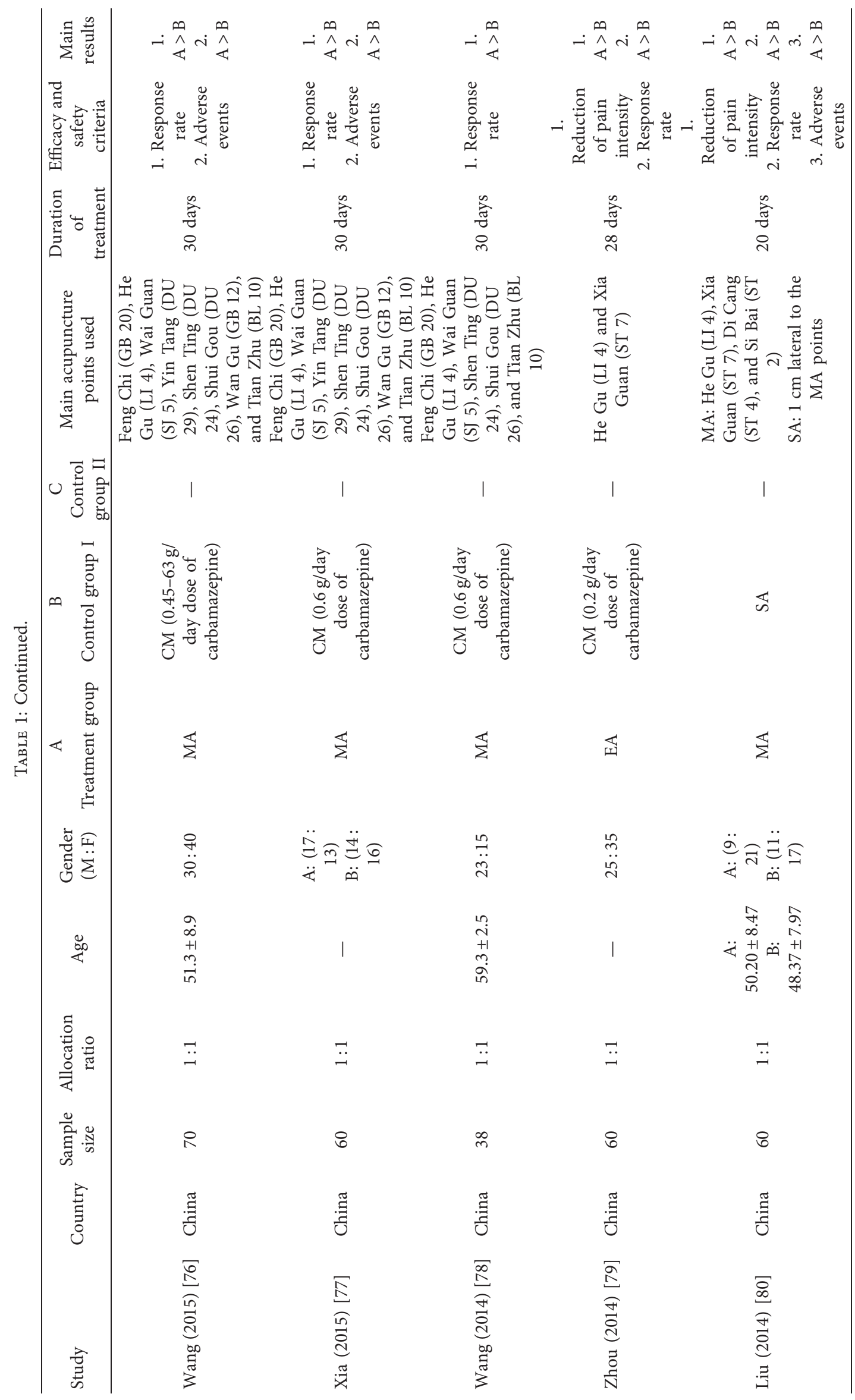




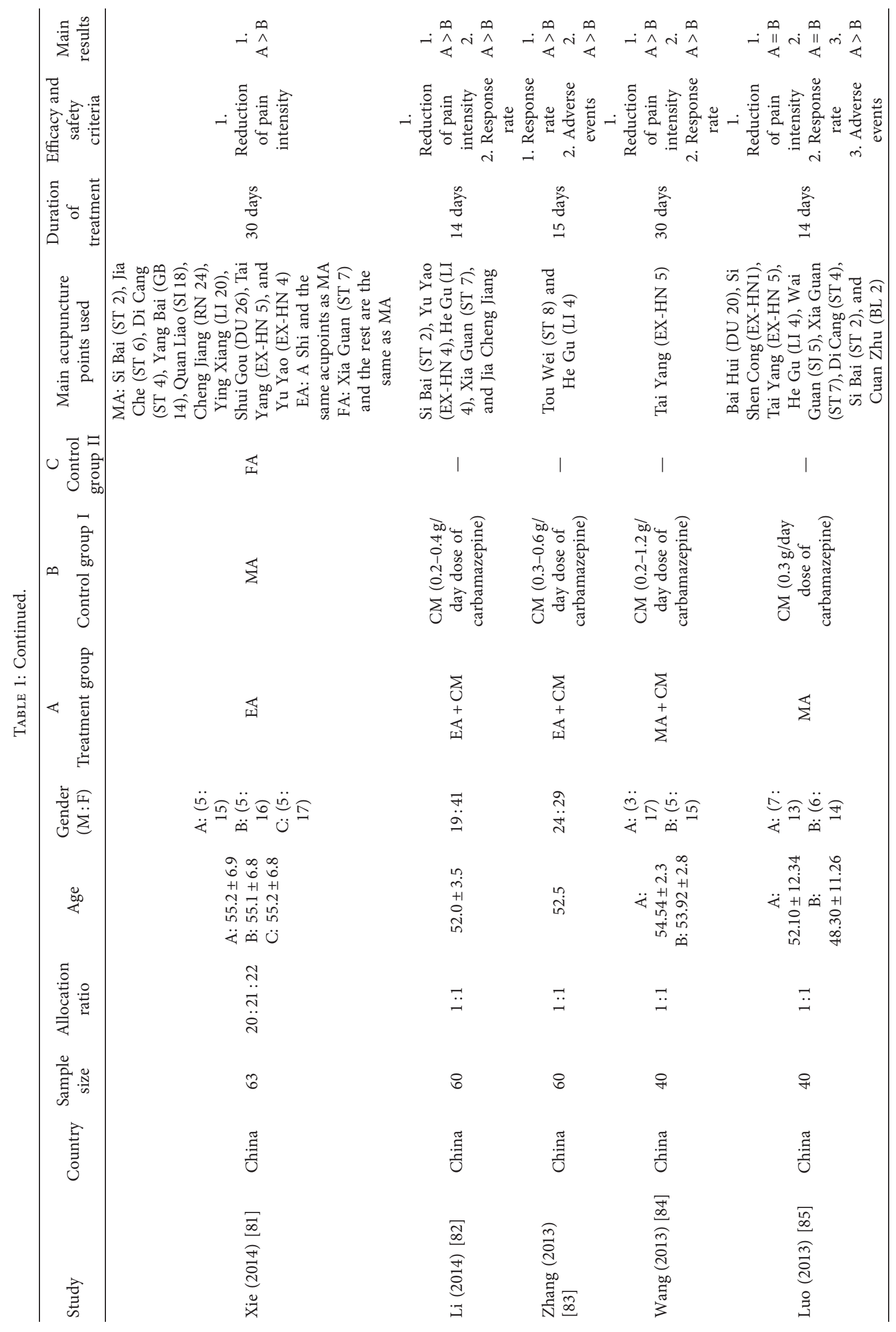




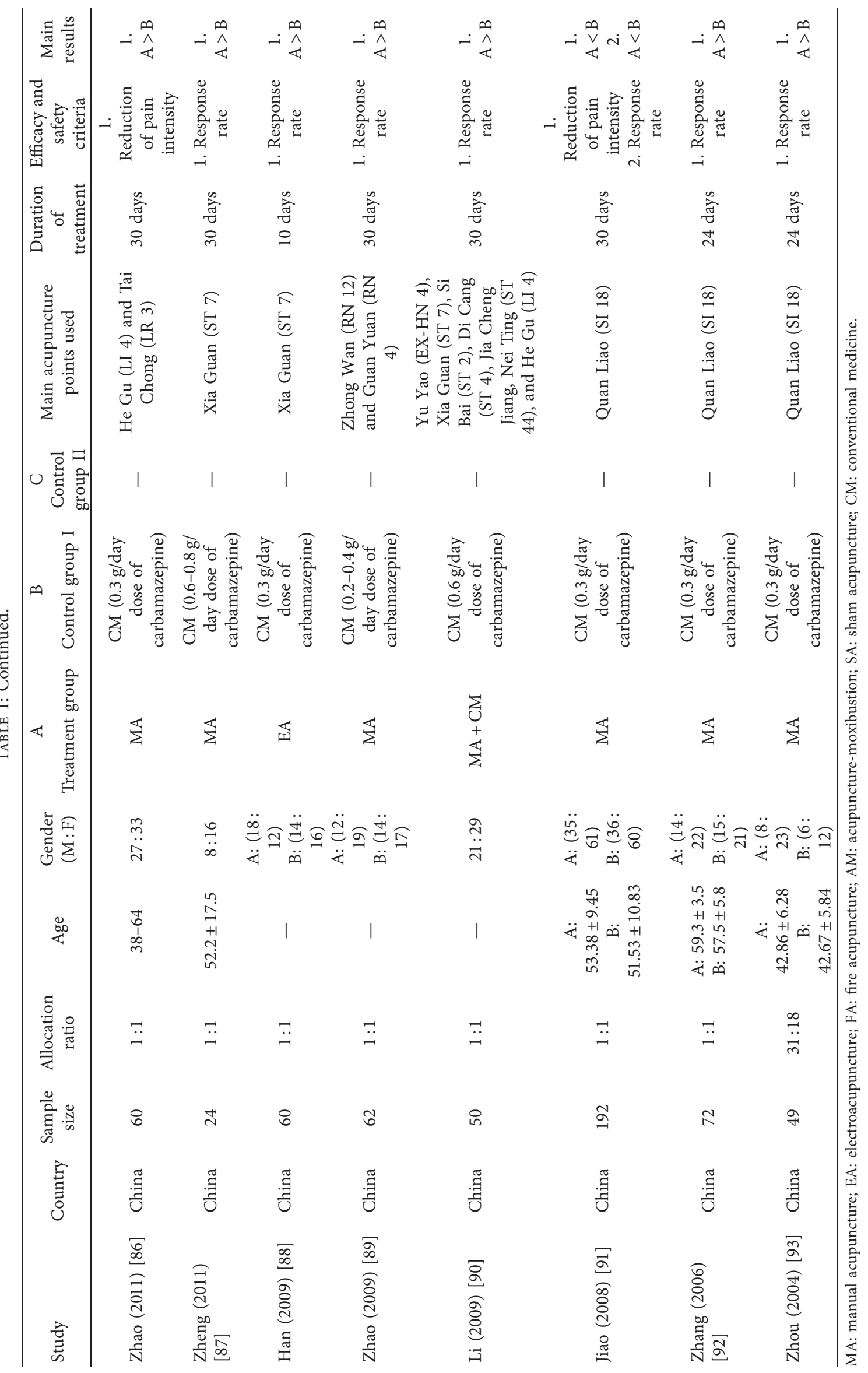




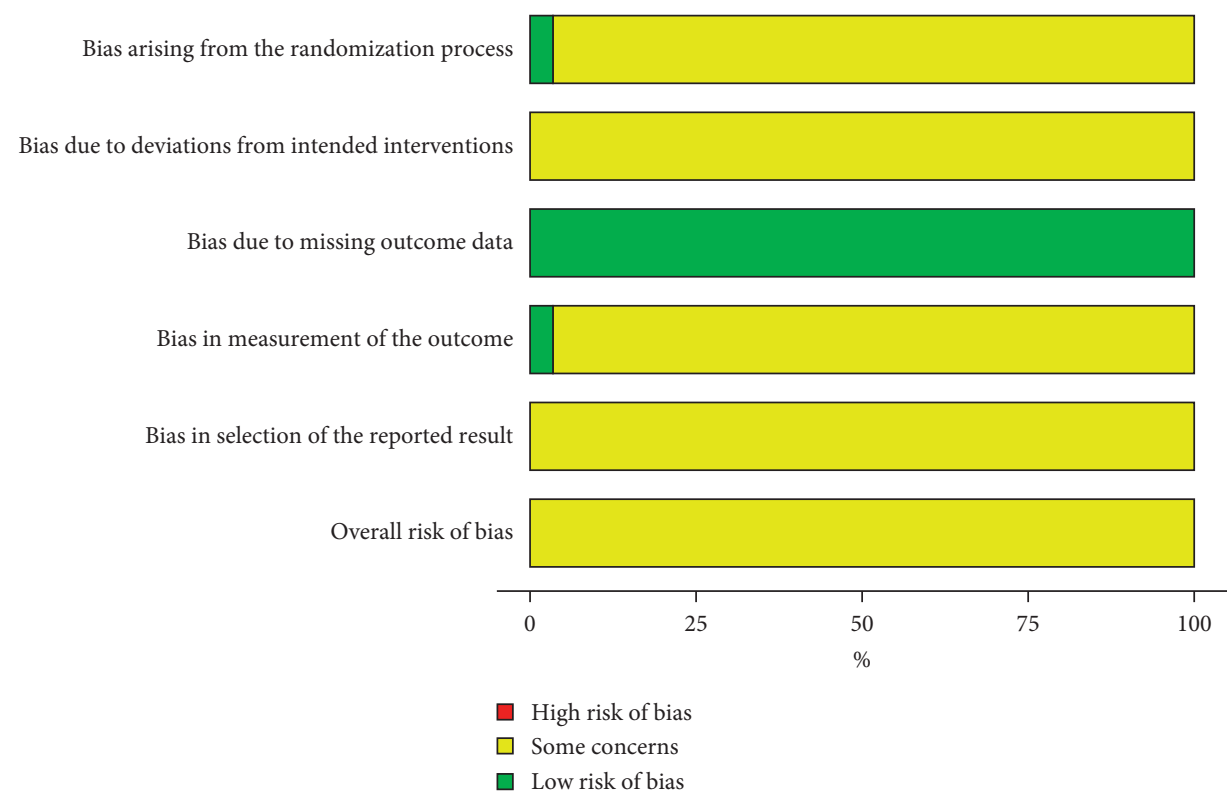

(a)

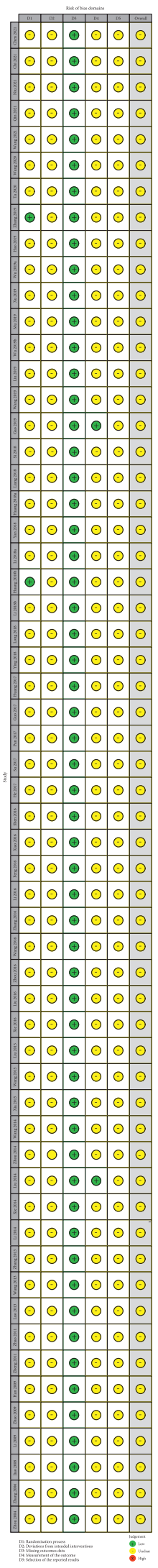

(b)

Figure 2: (a) Risk of bias graph; (b) risk of bias summary. 
TABLE 2: Pairwise meta-analysis of reduction of pain intensity.

\begin{tabular}{|c|c|c|c|c|c|}
\hline \multicolumn{2}{|c|}{ Comparison } & Number & MD (95\% CI) & $I^{2}$ & $p$ \\
\hline MA & $\mathrm{CM}$ & 9 & $1.14(0.48,1.80)^{*}$ & $89 \%$ & $<0.00001$ \\
\hline MA & $\mathrm{AM}$ & 1 & $-0.90(-1.87,0.07)$ & 一 & - \\
\hline MA & FA & 2 & $-0.84(-3.43,1.75)$ & $86 \%$ & 0.008 \\
\hline MA & SA & 1 & $1.66(0.93,2.39)^{*}$ & - & - \\
\hline EA & MA & 2 & $0.29(-1.49,2.07)$ & $71 \%$ & 0.06 \\
\hline EA & FA & 1 & $-1.40(-3.17,0.37)$ & - & - \\
\hline EA & $\mathrm{CM}$ & 1 & $1.07(0.09,2.05)^{*}$ & - & - \\
\hline $\mathrm{AM}$ & $\mathrm{CM}$ & 1 & $-0.08(-1.19,1.03)$ & - & - \\
\hline $\mathrm{MA}+\mathrm{EA}$ & MA & 1 & $3.18(2.43,3.93)^{*}$ & - & - \\
\hline $\mathrm{MA}+\mathrm{EA}$ & EA & 2 & $1.25(0.78,1.72)^{*}$ & $0 \%$ & 0.32 \\
\hline $\mathrm{MA}+\mathrm{CM}$ & $\mathrm{CM}$ & 7 & $1.19(0.55,1.84)^{*}$ & $89 \%$ & $<0.00001$ \\
\hline $\mathrm{AM}+\mathrm{CM}$ & $\mathrm{CM}$ & 3 & $1.88(0.87,2.90)^{*}$ & $85 \%$ & 0.001 \\
\hline $\mathrm{EA}+\mathrm{CM}$ & $\mathrm{CM}$ & 2 & $1.22(0.42,2.02)^{*}$ & $30 \%$ & 0.23 \\
\hline $\mathrm{EA}+\mathrm{CM}$ & $\mathrm{MA}+\mathrm{CM}$ & 2 & $0.61(-0.62,1.85)$ & $80 \%$ & 0.02 \\
\hline $\mathrm{MA}+\mathrm{CM}$ & $\mathrm{SA}+\mathrm{CM}$ & 1 & $1.60(0.32,2.88)^{*}$ & - & - \\
\hline
\end{tabular}

*Significant difference. MA: manual acupuncture; EA: electroacupuncture; AM: acupuncture-moxibustion; FA: fire acupuncture; SA: sham acupuncture; CM: conventional medicine.

TABle 3: Pairwise meta-analysis of response rate.

\begin{tabular}{|c|c|c|c|c|c|}
\hline \multicolumn{2}{|c|}{ Comparison } & \multirow{2}{*}{$\frac{\text { Number }}{25}$} & \multirow{2}{*}{ 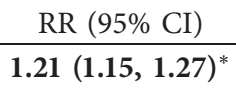 } & \multirow{2}{*}{$\frac{I^{2}}{44 \%}$} & \multirow{2}{*}{$\begin{array}{c}p \\
0.01\end{array}$} \\
\hline MA & $\mathrm{CM}$ & & & & \\
\hline MA & FA & 1 & $0.80(0.62,1.02)$ & - & - \\
\hline MA & $\mathrm{AM}$ & 1 & $0.89(0.74,1.08)$ & - & - \\
\hline MA & SA & 1 & $1.68(1.17,2.42)^{*}$ & - & - \\
\hline EA & MA & 1 & $1.12(0.93,1.35)$ & 一 & - \\
\hline EA & $\mathrm{CM}$ & 2 & $1.14(0.98,1.52)$ & 0 & 0.73 \\
\hline $\mathrm{AM}$ & $\mathrm{CM}$ & 1 & $1.08(0.84,1.40)$ & - & - \\
\hline $\mathrm{AM}+\mathrm{CM}$ & $\mathrm{CM}$ & 3 & $1.25(1.10,1.42)^{*}$ & $0 \%$ & 0.40 \\
\hline $\mathrm{MA}+\mathrm{CM}$ & $\mathrm{CM}$ & 10 & $1.20(1.14,1.26)^{*}$ & $0 \%$ & 0.68 \\
\hline $\mathrm{EA}+\mathrm{CM}$ & $\mathrm{CM}$ & 4 & $1.12(0.92,1.36)$ & $82 \%$ & 0.0008 \\
\hline $\mathrm{EA}+\mathrm{CM}$ & $\mathrm{MA}+\mathrm{CM}$ & 1 & $1.22(0.98,1.52)$ & - & - \\
\hline $\mathrm{MA}+\mathrm{EA}$ & MA & 1 & $1.50(1.13,1.99)^{*}$ & 一 & - \\
\hline $\mathrm{MA}+\mathrm{EA}$ & EA & 2 & $1.33(1.03,1.72)^{*}$ & 0 & 1 \\
\hline
\end{tabular}

${ }^{*}$ Significant difference. MA: manual acupuncture; EA: electroacupuncture; AM: acupuncture-moxibustion; FA: fire acupuncture; SA: sham acupuncture; $\mathrm{CM}$ : conventional medicine.

pain intensity reduction (Figure 3(a)), while 53 RCTs with 3,744 participants using 10 therapies showed a response rate (Figure 3(b)).

3.5.2. Statistical Inconsistency Analysis. The node-splitting method was used for local inconsistency analysis. The direct and indirect effects had no statistically different reduction of pain intensity and response rate $(p \geq 0.05)$.

3.5.3. Reduction in Pain Intensity. STATA 15.0 was used for network plot analysis of the 11 treatments (Figure 3(a). The Bayesian network meta-analysis was conducted using the consistency model to produce the ranking probability plot since the PSRF scores were close to 1 (Appendix 3(a)) and $p$ values were higher than 0.05 (Appendix 3(b)). MA + EA, FA, and $\mathrm{AM}+\mathrm{CM}$ significantly reduced pain intensity (Figure 4(a)). However, MA + EA was the optimum intervention method. The meta-analyses details are shown in Table 4. MA + EA was more effective than EA, MA, $\mathrm{MA}+\mathrm{CM}, \mathrm{AM}, \mathrm{CM}, \mathrm{SA}$, and $\mathrm{SA}+\mathrm{CM}$. Furthermore, $\mathrm{MA}+\mathrm{EA}$ and FA were more effective than MA, SA + CM, $\mathrm{SA}$, and CM. MA + EA, FA, AM+ CM, EA, EA + CM, MA, and $\mathrm{MA}+\mathrm{CM}$ were more effective than $\mathrm{CM}$. AM + CM was significantly effective than SA.

3.5.4. Response Rate. STATA 15.0 was also used for network plot analysis of 10 interventions (Figure 3(b)). Similarly, the Bayesian meta-analysis was conducted using the consistency model to produce the ranking probability plot (Figure 4(b)) since the PSRF scores were close to 1 (Appendix 4(a)) and $p$ values were higher than 0.05 (Appendix 4(b)). MA + EA, FA, and AM had the most significant response rates (Figure 4(b)). However, $\mathrm{MA}+\mathrm{EA} / \mathrm{FA}$ maybe the optimum intervention method. The meta-analyses details are shown in Table 5. $\mathrm{MA}+\mathrm{EA}$ was more effective than 4 interventions (EA, MA, CM, and SA). $\mathrm{MA}+\mathrm{EA}, \mathrm{MA}+\mathrm{CM}, \mathrm{MA}, \mathrm{EA}+\mathrm{CM}$, and $\mathrm{AM}+\mathrm{CM}$ were more effective than $\mathrm{CM}$, and $\mathrm{MA}+\mathrm{EA}, \mathrm{FA}, \mathrm{AM}, \mathrm{EA}, \mathrm{MA}$, $\mathrm{EA}+\mathrm{CM}$, and $\mathrm{AM}+\mathrm{CM}$ were highly effective than $\mathrm{SA}$.

3.6. Safety. A total of 24 studies using MA, CM, SA, $\mathrm{EA}+\mathrm{CM}, \mathrm{AM}+\mathrm{CM}$, and $\mathrm{MA}+\mathrm{CM}$ demonstrated adverse effects, with 14 studies indicating 25 MA-related adverse events. Besides, 22 studies described 205 CM-related adverse events and one study showed one SA-related adverse event. Three studies reported $52 \mathrm{MA}+\mathrm{CM}$-related adverse events, one study showed seven EA + CM-related adverse events, and one study reported two $\mathrm{AM}+\mathrm{CM}$-related adverse events. The details of adverse events between acupuncture and the CM group are shown in Table 6.

3.7. Heterogeneity. RevMan 5.3 was used for sensitivity analysis to evaluate the stability and reliability of the results. The results were shown to be considerably reliable and stable 


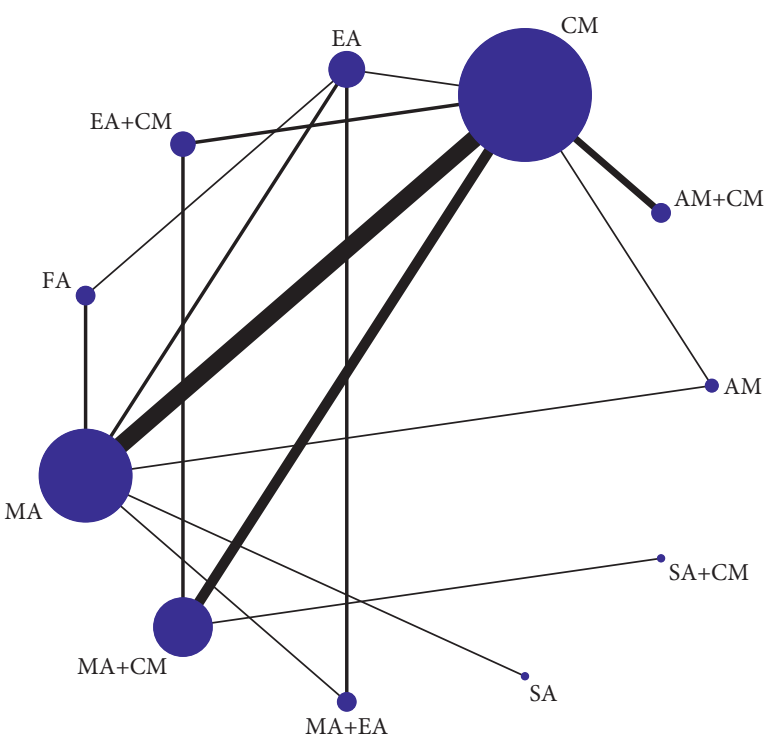

(a)

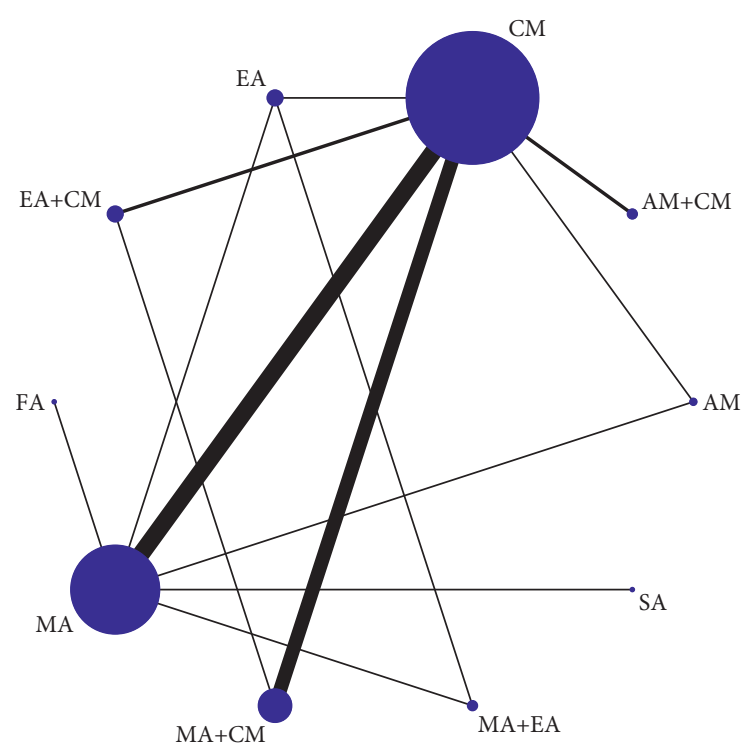

(b)

Figure 3: The network graph of different interventions of (a) pain relief and (b) response rate.

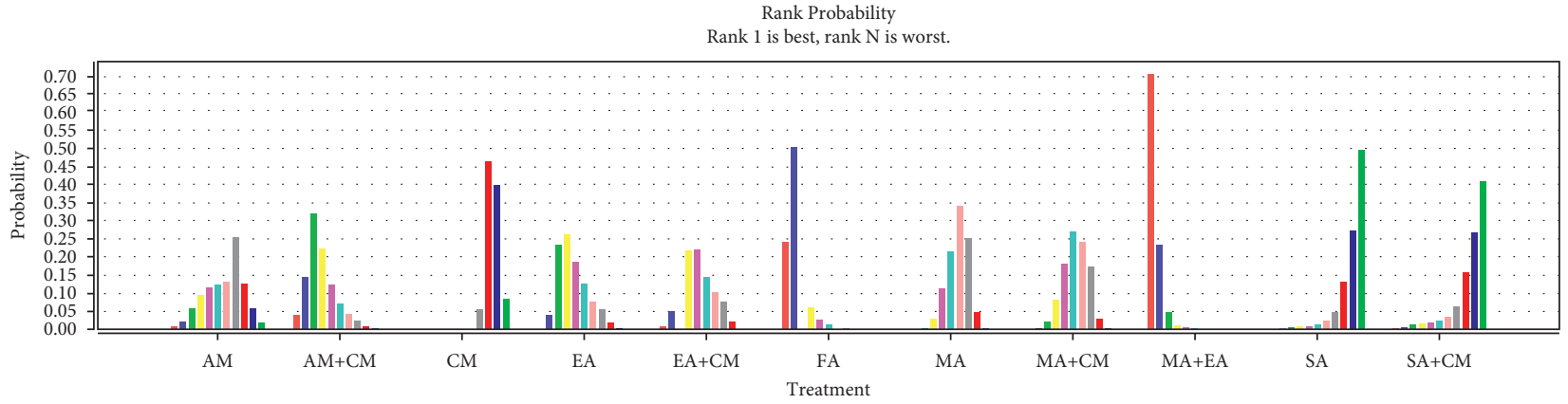

- Rank 1

- Rank 2

- Rank 3

- Rank 4

- Rank 5

Rank 6

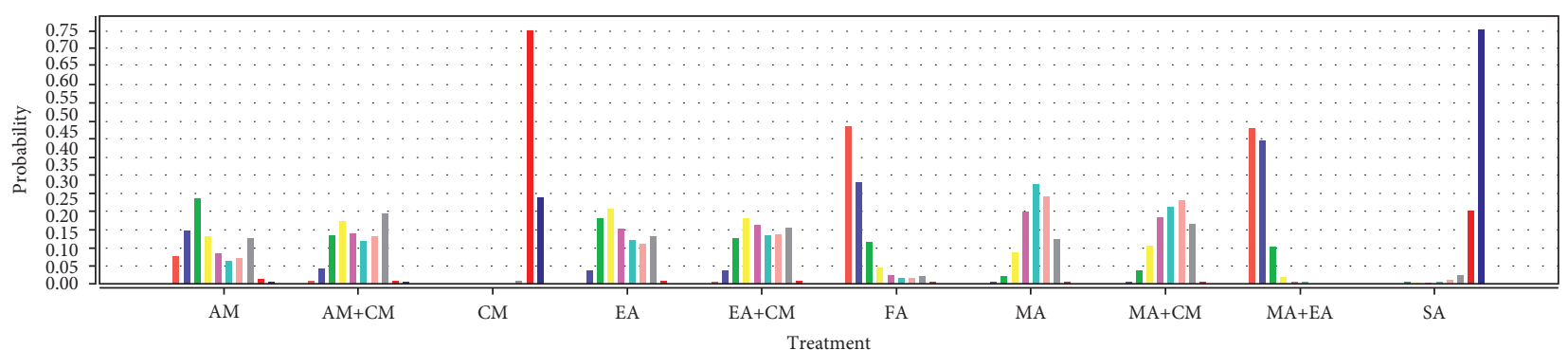

- Rank 1

- Rank 2

- Rank 3

- Rank 4

- Rank 5 (a)

Rank Probability

Rank 1 is best, rank $\mathrm{N}$ is worst.

-1 Rank 7

- Rank 8

- Rank 9

- Rank 10

- Rank 11

Treatment

- Rank 6

- Rank 7

- Rank 8

- Rank 9

- Rank 10

(b)

FIGURE 4: The figure of ranking probability of (a) pain relief and (b) response rate. 


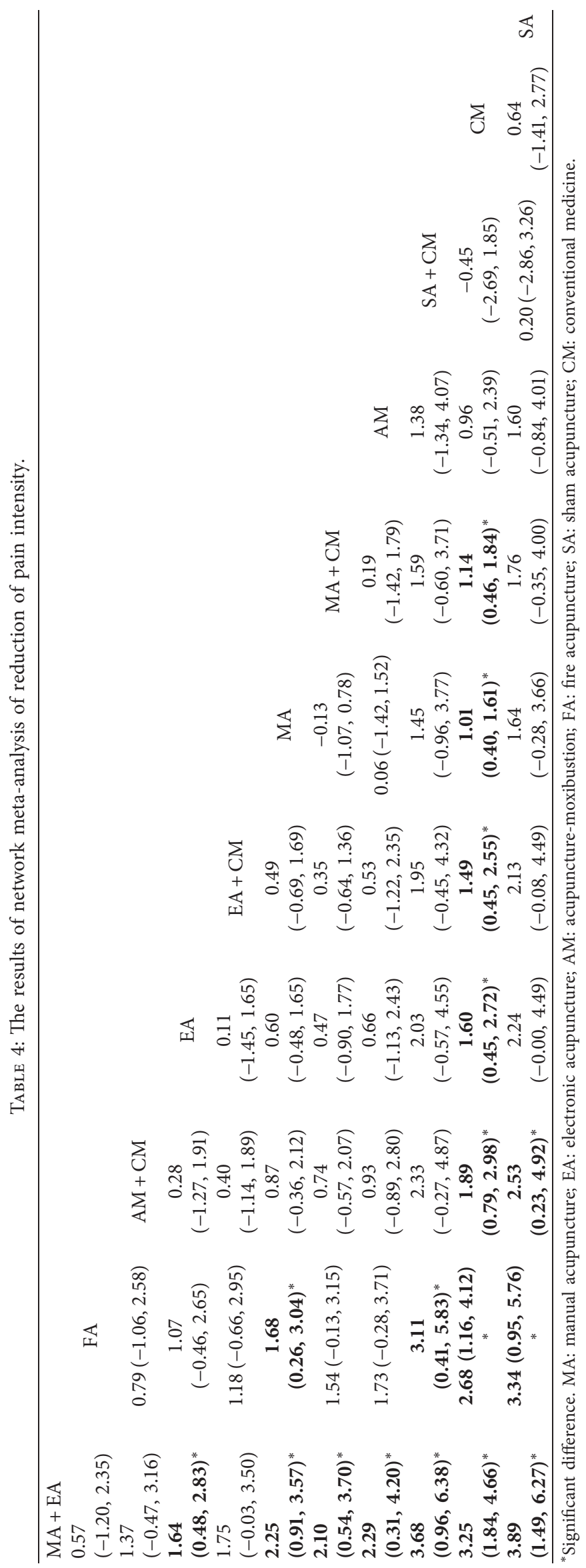




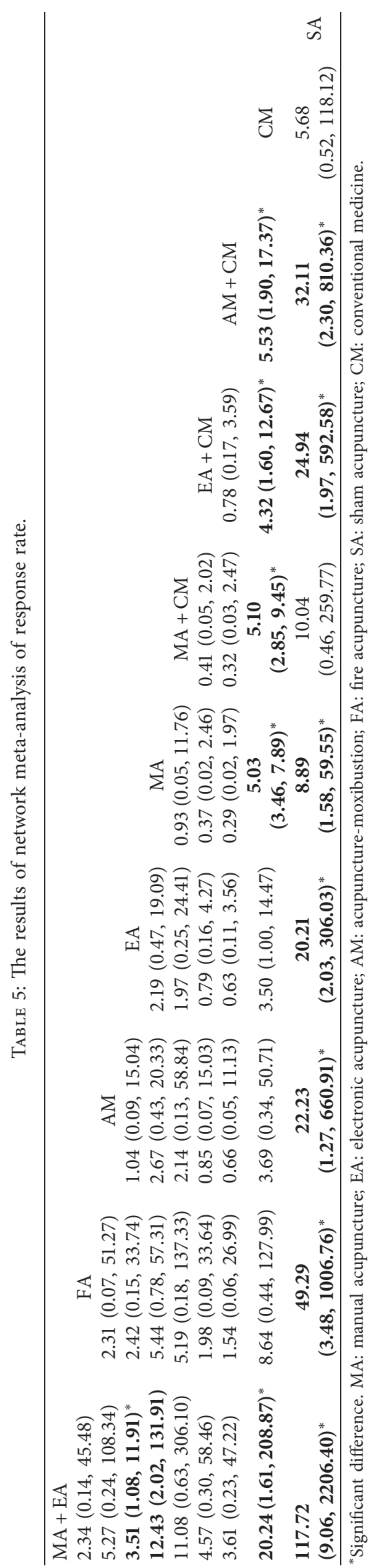


TABLE 6: Adverse events in included RCTs.

\begin{tabular}{|c|c|c|c|}
\hline Interventions & Study (reference) & $\begin{array}{l}\text { Sample } \\
\text { size }\end{array}$ & Adverse events \\
\hline \multirow{12}{*}{ MA } & Chen (2021) [38] & 40 & 1 case \\
\hline & Zhang (2019) [45] & 33 & 1 case of fatigue; 1 case of dizziness; 1 case of drowsiness \\
\hline & Liu (2019) [51] & 44 & 1 case \\
\hline & Huang (2018a) [55] & 32 & 1 case of hyperpigmentation \\
\hline & Yan (2018) [56] & 36 & $\begin{array}{c}1 \text { case of dizziness; } 1 \text { case of gastrointestinal reaction; } 1 \text { case of fatigue; } 1 \text { case of rash; } 1 \\
\text { case of pruritus }\end{array}$ \\
\hline & Li (2018b) [59] & 44 & 1 case of nausea and vomiting \\
\hline & Xiao (2016) [67] & 50 & 1 case of dizziness \\
\hline & Li (2016) [69] & 25 & 1 case \\
\hline & Zhou (2016) [72] & 32 & 1 case \\
\hline & Liu (2016) [73] & 30 & 1 case of dizziness; 1 case of fatigue \\
\hline & Xie (2016) [74] & 40 & 2 cases \\
\hline & Liu (2015) [75] & 42 & 3 cases of drowsiness \\
\hline \multirow{24}{*}{$\mathrm{CM}$} & Wang (2015) [76] & 35 & 2 cases \\
\hline & Xia (2015) [77] & 30 & 1 case \\
\hline & Chen (2021) [38] & 40 & 6 cases \\
\hline & Chi (2021) [39] & 39 & 2 cases of drowsiness; 3 cases of dizziness; 3 cases of nausea and vomiting \\
\hline & Zhang (2019) [45] & 29 & $\begin{array}{c}2 \text { cases of fatigue; } 1 \text { case of dizziness; } 2 \text { cases of drowsiness; } 2 \text { cases of gastrointestinal } \\
\text { reaction }\end{array}$ \\
\hline & $\mathrm{Xu}(2019)[48]$ & 33 & 1 case of dizziness; 1 case of drowsiness; 2 cases of nausea \\
\hline & Liu (2019) [51] & 44 & 6 cases \\
\hline & Si (2018) [53] & 33 & $\begin{array}{c}2 \text { cases of peripheral facial paralysis; } 4 \text { cases of facial numbness; } 5 \text { cases of tinnitus; } 6 \text { cases } \\
\text { of herpesvirus infection }\end{array}$ \\
\hline & Liang (2018) [54] & 43 & $\begin{array}{c}1 \text { case of dizziness; } 2 \text { cases of nausea and vomiting; } 3 \text { cases of drowsiness; } 1 \text { case of fever; } 1 \\
\text { case of pruritus }\end{array}$ \\
\hline & Huang (2018a) [55] & 31 & 1 case of dermatitis; 5 cases of drowsiness \\
\hline & Yan (2018) [56] & 36 & $\begin{array}{c}3 \text { cases of dizziness; } 4 \text { cases of gastrointestinal reaction; } 1 \text { case of fatigue; } 2 \text { cases of rash; } 3 \\
\text { cases of pruritus }\end{array}$ \\
\hline & Li (2018b) [59] & 44 & 1 case of rash; 2 cases of pruritus; 1 case of dizziness; 3 cases of fatigue \\
\hline & Shen $(2016)[66]$ & 40 & 1 case of dermatitis; 6 cases of dizziness \\
\hline & Xiao (2016) [67] & 50 & 6 cases of gastrointestinal reaction; 4 cases of abnormal liver function; 2 cases of rash \\
\hline & $\operatorname{Li}(2016)[69]$ & 25 & 5 cases \\
\hline & Zhou (2016) [72] & 32 & 6 cases \\
\hline & Liu (2016) [73] & 30 & 1 case of dizziness; 1 case of gastrointestinal reaction; 2 cases of fatigue; 1 case of nausea \\
\hline & Xie (2016) [74] & 40 & 8 cases \\
\hline & Liu (2015) [75] & 42 & 3 cases of dizziness; 5 cases of drowsiness; 3 cases of nausea \\
\hline & Wang (2015) [76] & 35 & 8 cases \\
\hline & Xia (2015) [77] & 30 & 5 cases \\
\hline & Zhang (2013) [83] & 28 & 5 cases of dizziness; 6 cases of gastrointestinal reaction \\
\hline & Luo (2013) [85] & 20 & $\begin{array}{l}3 \text { cases of dizziness; } 5 \text { cases of drowsiness; } 1 \text { case of xerostomia; } 2 \text { cases of nausea and } \\
\text { vomiting; } 2 \text { cases of anorexia; } 2 \text { cases of skin disorders }\end{array}$ \\
\hline & Zhao (2009) [89] & 20 & $\begin{array}{c}1 \text { case of dizziness; } 3 \text { cases of drowsiness; } 3 \text { cases of xerostomia; } 4 \text { cases of nausea and } \\
\text { vomiting; } 3 \text { cases of anorexia; } 6 \text { cases of constipation; } 1 \text { case of abnormal liver function; } 3 \\
\text { cases of abnormal blood; } 1 \text { case of abnormal renal function; } 2 \text { cases of restless; } 8 \text { cases of } \\
\text { skin disorders }\end{array}$ \\
\hline \multirow{3}{*}{ SA } & Liu (2014) [80] & 28 & 1 case of dizziness \\
\hline & Chi (2021) [39] & 39 & 1 case of dizziness; 1 case of nausea and vomiting \\
\hline & Shen (2016) [66] & 40 & 2 cases of hyperpigmentation \\
\hline $\mathrm{MA}+\mathrm{CM}$ & Zhao (2009) [89] & 20 & $\begin{array}{c}4 \text { cases of dizziness; } 7 \text { cases of drowsiness; } 6 \text { cases of xerostomia; } 1 \text { case of nausea and } \\
\text { vomiting; } 12 \text { cases of anorexia; } 6 \text { cases of constipation; } 3 \text { cases of abnormal liver function; } \\
2 \text { cases of abnormal blood; } 2 \text { cases of abnormal renal function; } 3 \text { cases of restless; } 2 \text { cases of } \\
\text { skin disorders }\end{array}$ \\
\hline $\mathrm{EA}+\mathrm{CM}$ & Si (2018) [53] & 33 & $\begin{array}{c}1 \text { case of peripheral facial paralysis; } 1 \text { case of facial numbness; } 3 \text { cases of tinnitus; } 2 \text { cases of } \\
\text { herpesvirus infection }\end{array}$ \\
\hline $\mathrm{AM}+\mathrm{CM}$ & $\mathrm{Xu}(2019)[48]$ & 33 & 2 cases of dizziness \\
\hline
\end{tabular}

MA: manual acupuncture; EA: electronic acupuncture; SA: sham acupuncture; AM: acupuncture-moxibustion; CM: conventional medicine. 


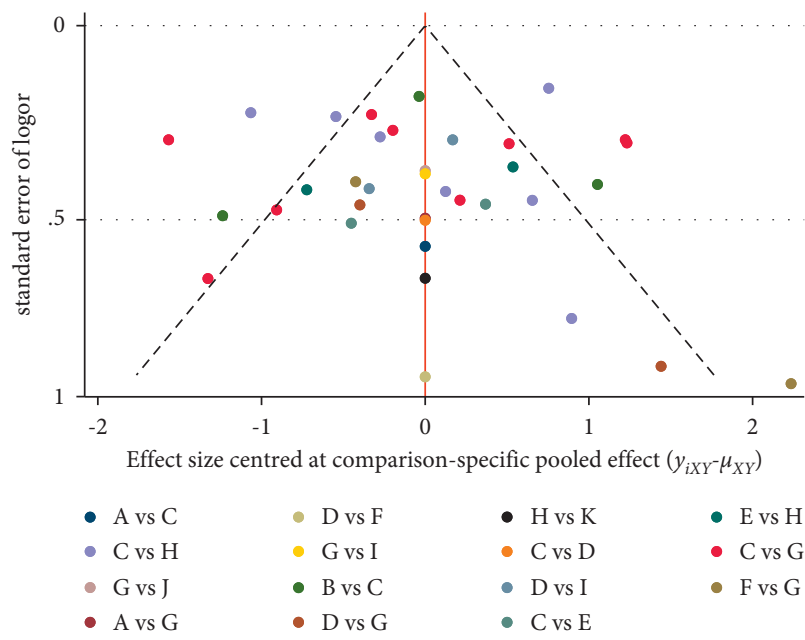

(a)

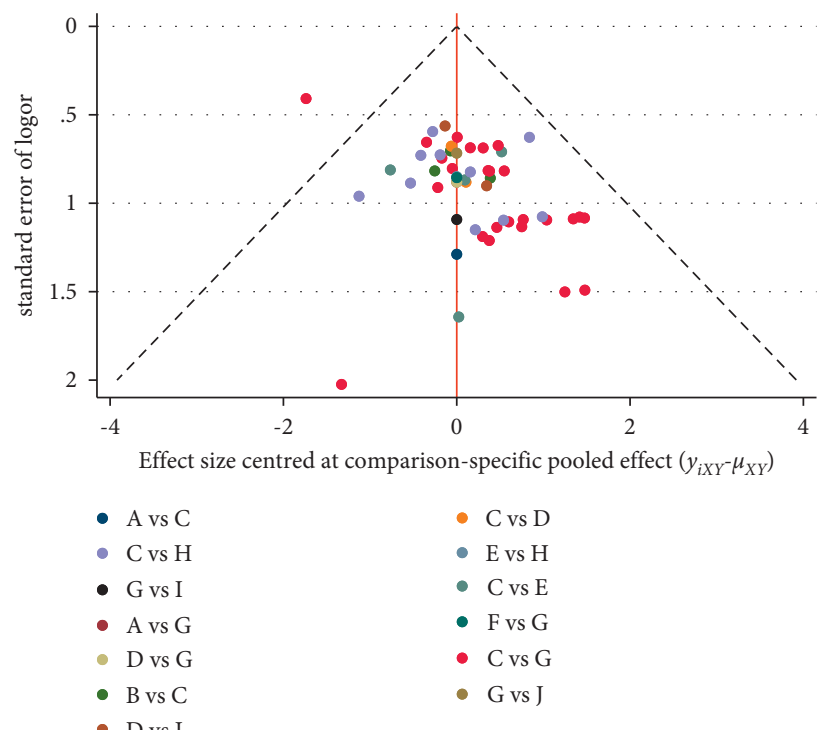

(b)

FiguRE 5: (a) Funnel plot for the network meta-analysis of pain relief. (b) Funnel plot for the network meta-analysis of response rate. Note. A: AM; B: AM+ CM; C: CM; D: EA; E: EA + CM; F: FA; G: MA; H: MA+ CM; I: MA+ EA; J: SA; K: SA +CM.

and could have high clinical heterogeneity due to the diverse selection of acupoints, duration of treatment, carbamazepine dose, and the like.

3.8. Publication Bias. A funnel graph was used to determine the reporting bias assessment (Figure 5). The graph indicated a low risk of publication bias, as shown in the comparisonadjusted funnel plots for reducing pain intensity (Figure 5(a)). However, the funnel plots of response rate indicated a potential reporting bias (Figure 5(b)).

3.9. Quality of Evidence. The GRADE criteria were used to compare the direct and indirect evidence. The quality of outcomes was critically low to moderate. However, most evidence were critically low (Appendixes 5 and 6), mainly due to the ROB, inconsistency, and imprecision.

\section{Discussion}

Although CM (carbamazepine) is used for PTN treatment, it has some inevitable adverse effects [94]. In this study, alternative PTN treatments were explored. Acupuncture has been successfully adopted for PTN treatment for a long time. However, several acupuncture therapies used are not regulated and standardized. NMA is used when there is no possibility of head-to-head or direct comparison of the intervention of interest versus control. Thus, it is applied to analyze several RCTs with various acupuncture therapies and rank these interventions [95]. This is the first study to conduct a Bayesian network meta-analysis to assess the efficacy of different acupuncture methods for PTN treatment based on the PRISMA-NMA.
4.1. Summary of Evidence. In this study, the efficacy of various therapies for PTN treatment was compared and reliable results were obtained [96, 97]. For reduction of pain intensity, EA + MA efficacy was statistically different compared with EA and MA efficacies. MA/EA efficacy was also statistically significant compared with CM efficacy. Furthermore, MA/EA/ AM combined with CM showed statistically significant efficacy compared with CM. FA and SA also had different efficacies. EA + MA was the optimal acupuncture method for pain intensity reduction. EA + MA significantly reduced pain intensity compared with EA, MA, MA+CM, AM, CM, SA, and $\mathrm{SA}+\mathrm{CM} . \mathrm{MA}+\mathrm{EA}, \mathrm{FA}, \mathrm{AM}+\mathrm{CM}, \mathrm{EA}, \mathrm{EA}+\mathrm{CM}, \mathrm{MA}$, and $\mathrm{MA}+\mathrm{CM}$ were more effective than CM. For the response rate, $\mathrm{MA}$ and $\mathrm{CM}$ had different efficacies. $\mathrm{MA}+\mathrm{EA}, \mathrm{MA}+\mathrm{CM}, \mathrm{MA}$, $\mathrm{EA}+\mathrm{CM}$, and $\mathrm{AM}+\mathrm{CM}$ were more effective than $\mathrm{CM}$. $\mathrm{MA}+\mathrm{EA}, \mathrm{FA}, \mathrm{AM}, \mathrm{EA}, \mathrm{MA}, \mathrm{EA}+\mathrm{CM}$, and $\mathrm{AM}+\mathrm{CM}$ were highly effective than SA. EA + MA/FA was the optimal acupuncture treatment for improved response rate. However, most evidence had a critically low quality. In safety, 24 RCTs (41.38\%) showed adverse events and 22 RCTs (37.93\%) reported carbamazepine-related adverse events (dizziness, drowsiness, and gastrointestinal reaction). However, 15 RCTs (25.86\%) reported acceptable acupuncture-related adverse events (dizziness, drowsiness, and fatigue). No study reported severe adverse events.

4.2. Strengths and Limitations. This study has several strengths. (a) This is the first network meta-analysis to include 58 RCTs and compare different acupuncture methods, such as AM, EA, FA, MA, and their combinations with CM, or acupuncture versus sham acupuncture and CM groups for PTN treatment; (b) this systematic review was registered on PROSPERO and followed the PRISMA-NMA guidelines to reduce ROB; (c) ICC was 
used to assess the reviewers' agreements to ensure the reliability of the assessment results; (d) GRADE was used to assess the quality of evidence.

However, this study has some limitations. First, the evidences were of low quality, and several limitations, including study design, implementation, analysis, and publication, influence ROB. Second, while there were strict criteria to control the quality of evidences, the included studies had several therapies and can affect the evidence quality. Third, the results had a statistically significant heterogeneity possibly due to age, gender, treatments, details of acupuncture, carbamazepine dose, or other factors from the PTN patients. Besides, most included RCTs had no follow-up time and the long-term effect of acupuncture could not be determined. Finally, all the included studies were from China and the search strategies were limited to English and Chinese, thus regional limitation.

4.3. Suggestions to Future Research. In this study, the methodological quality of all trials was moderate, but the quality of evidences was critically low, possibly due to the exclusion of several details (randomization, deviations from intended interventions, outcome measurement, and selective bias). Therefore, the project design, implementation, analysis, and writing of studies should strictly follow the latest edition of Cochrane Handbook for Systematic Reviews [98], the Consolidated Standards of Reporting Trials (CONSORT) [99], and STRICTA [27] in the future. Second, this study did not show the long-term efficacy and safety of acupuncture methods. Therefore, it is important to consider longitudinal trials to explore long-term efficacy and safety. Moreover, several factors, such as acupoint selection, treatment duration, intervention time, and dose of carbamazepine, impact heterogeneity. Therefore, future studies should standardize and regulate the acupuncture and CM details for PTN treatment. Finally, other core outcomes, such as changes in the psychological situation and quality of life, should be considered [100].

\section{Conclusion}

In conclusion, acupuncture reduces pain intensity, improves response rate, and has less adverse events on PTN patients. Moreover, five acupuncture therapies are superior to conventional medicine (carbamazepine). In this study, EA + MA was regarded as the optimal therapy for PTN. However, the overall quality of evidences from included studies was critically low. Therefore, well-designed and high-quality clinical trials are needed to confirm the abovementioned findings in the future.

\section{Abbreviations}

ADDIS: Aggregate Data Drug Information System

AE: $\quad$ Adverse event

AM: $\quad$ Acupuncture-moxibustion

AMED: $\quad$ Allied and Complementary Medicine

Database
CBM: $\quad$ Chinese Biomedical Literature Database

CENTRAL: Cochrane Central Register of Controlled Trials

ChiCTR: Clinical Trials and Chinese Clinical Trial Register

CI: $\quad$ Confidence interval

CM: $\quad$ Conventional medicine

CNKI: China National Knowledge Infrastructure

CONSORT: Consolidated Standards of Reporting Trials

EA: $\quad$ Electronic acupuncture

FA: $\quad$ Fire acupuncture

GRADE: Grades of Recommendations, Assessment, Development, and Evaluation

ICC: Intraclass correlation coefficient

MA: $\quad$ Manual acupuncture

MCMC: Markov Chain Monte Carlo

MD: $\quad$ Mean difference

NMA: $\quad$ Network meta-analysis

NRS: $\quad$ Numerical rating scale

PICOS: Participant, intervention, control, outcome, and study design

PRISMA: Preferred Reporting Items for Systematic

Reviews and Meta-Analyses

PSRF: $\quad$ Potential scale reduced factor

PTN: $\quad$ Primary trigeminal neuralgia

RCTs: $\quad$ Randomized controlled trials

RevMan: Review Manager

ROB: $\quad$ Risk of bias

RR: $\quad$ Relative risk

SA: $\quad$ Sham acupuncture

SP: $\quad$ Substance P

STRICTA: Standards for Reporting Interventions in Clinical Trials of Acupuncture

VAS: Visual analogue scale

VIP: China Science and Technology Journal Database

WF: $\quad$ Wanfang Database

WHO World Health Organization International

ICTRP: Clinical Trials Registry Platform

WOS: $\quad$ Web of Science.

\section{Data Availability}

The data used to support the findings of this systematic review are included within the study.

\section{Conflicts of Interest}

The authors declare that there are no conflicts of interest.

\section{Authors' Contributions}

Zihan Yin and Fanrong Liang conceived this study. Zihan Yin developed and implemented the systematic review, under the supervision of Ling Zhao. Zihan Yin and Mingsheng Sun provided the statistical analysis plan for the study and performed data analysis. Zihan Yin and Fumin Wang performed study search, screening, and extraction of data, whereas Fanrong Liang and Ling Zhao reviewed the work. Zihan Yin wrote the first draft of the current review, 
and Fanrong Liang and Ling Zhao provided input to the final draft. All authors read and approved the final manuscript.

\section{Acknowledgments}

This work was financially supported by the National Key Research and Development Program of China (No. 2019YFC1709700), the National Natural Science Foundation of China (Nos. 81590951, 82004486, and 81973961), and the Project of Science and Technology Department of Sichuan Province (Nos. 20ZDYF1199 and 2019YFS0081).

\section{Supplementary Materials}

Appendix 1: PRISMA-NMA statement reporting standard checklist. Appendix 2: search strategies of each database. Appendix 3: (a) the PSRF value of reduction in pain intensity and (b) node-splitting test result of reduction in pain intensity. Appendix 4: (a) the PSRF value of response rate and (b) node-splitting test result of response rate. Appendix 5: quality of evidence of reduction in pain intensity in network meta-analysis. Appendix 6: quality of evidence of response rate in network meta-analysis. (Supplementary Materials)

\section{References}

[1] L. Bendtsen, J. M. Zakrzewska, J. Abbott et al., "European Academy of Neurology guideline on trigeminal neuralgia," European Journal of Neurology, vol. 26, no. 6, pp. 831-849, 2019.

[2] S. Maarbjerg, G. Di Stefano, L. Bendtsen, and G. Cruccu, "Trigeminal neuralgia-diagnosis and treatment," Cephalalgia, vol. 37, no. 7, pp. 648-657, 2017.

[3] H. Hu, L. Chen, R. Ma, H. Gao, and J. Fang, "Acupuncture for primary trigeminal neuralgia: a systematic review and PRISMA-compliant meta-analysis," Complementary Therapies in Clinical Practice, vol. 34, pp. 254-267, 2019.

[4] T. Tölle, E. Dukes, and A. Sadosky, "Patient burden of trigeminal neuralgia: results from a cross-sectional survey of health state impairment and treatment patterns in six European countries," Pain Practice: The Official Journal of World Institute of Pain, vol. 6, no. 3, pp. 153-160, 2006.

[5] K. W. Al-Quliti, "Update on neuropathic pain treatment for trigeminal neuralgia. The pharmacological and surgical options," Neurosciences, vol. 20, no. 2, pp. 107-114, 2015.

[6] J. S. Koopman, J. P. Dieleman, F. J. Huygen, M. de Mos, C. G. Martin, and M. C. Sturkenboom, "Incidence of facial pain in the general population," Pain, vol. 147, no. 1-3, pp. 122-127, 2009.

[7] S. Maarbjerg, A. Gozalov, J. Olesen, and L. Bendtsen, "Trigeminal neuralgia--a prospective systematic study of clinical characteristics in 158 patients," Headache: The Journal of Head and Face Pain, vol. 54, no. 10, pp. 1574-1582, 2014.

[8] P. Asplund, P. Blomstedt, and A. T. Bergenheim, "Percutaneous balloon compression vs percutaneous retrogasserian glycerol rhizotomy for the primary treatment of trigeminal neuralgia," Neurosurgery, vol. 78, no. 3, pp. 421-428, 2016.

[9] E. I. Araya, R. F. Claudino, E. J. Piovesan, and J. G. Chichorro, "Trigeminal neuralgia: basic and clinical aspects," Current Neuropharmacology, vol. 18, no. 2, pp. 109-119, 2020.
[10] B. Chen, H. Choi, L. J. Hirsch et al., "Psychiatric and behavioral side effects of antiepileptic drugs in adults with epilepsy," Epilepsy and Behavior, vol. 76, pp. 24-31, 2017.

[11] Z. Huang, J. Huo, and J. Zhao, "[Efficacy on primary trigeminal neuralgia treated with triple puncture technique and electroacupuncture at trigger points]," Zhongguo Zhen Jiu, vol. 37, no. 1, pp. 31-34, 2017.

[12] Y. Fu, H. F. Zhang, F. Li et al., "[Comparative research of moxibustion and infrared method in testing heat-sensitive state at Xiaguan (ST 7) in primary trigeminal neuralgia]," Zhongguo Zhen Jiu, vol. 33, no. 5, pp. 411-414, 2013.

[13] Y. Fu, H. F. Zhang, F. Li et al., "[Observation on the distribution of heat-sensitized acupoints in patients with primary trigeminal neuralgia]," Zhongguo Zhen Jiu, vol. 33, no. 4, pp. 325-327, 2013.

[14] L. He, W. Y. Zhou, and X. M. Zhang, "[Trigeminal neuralgia of hyperactive of liver yang type treated with acupuncture at Xiaguan (ST 7) at different depth: a randomized controlled trial]," Zhongguo Zhen Jiu, vol. 32, no. 2, pp. 107-110, 2012.

[15] X. Zhu, "Electro-acupuncture combined with the trigger point needle-embedding for treatment of primary trigeminal neuralgia in 31 cases," Journal of traditional Chinese medicine, vol. 28, no. 1, pp. 13-14, 2008.

[16] M. C. Ichida, M. Zemuner, J. Hosomi et al., "Acupuncture treatment for idiopathic trigeminal neuralgia: a longitudinal case-control double blinded study," Chinese Journal of Integrative Medicine, vol. 23, no. 11, pp. 829-836, 2017.

[17] J. Gao, C. Zhao, W. Jiang, B. Zheng, and Y. He, "Effect of acupuncture on cognitive function and quality of life in patients with idiopathic trigeminal neuralgia," The Journal of Nervous and Mental Disease, vol. 207, no. 3, pp. 171-174, 2019.

[18] I. S. Lee, S. Cheon, and J. Y. Park, "Central and peripheral mechanism of acupuncture analgesia on visceral pain: a systematic review," Evidence-based Complementary and Alternative Medicine, vol. 2019, Article ID 1304152, 2019.

[19] H. V. Acar, "Acupuncture and related techniques during perioperative period: a literature review," Complementary Therapies in Medicine, vol. 29, pp. 48-55, 2016.

[20] L. Qiao, M. Guo, J. Qian, B. Xu, C. Gu, and Y. Yang, "Research advances on acupuncture analgesia," The American Journal of Chinese Medicine, vol. 48, no. 2, pp. 245-258, 2020.

[21] T. Chen, W. W. Zhang, Y. X. Chu, and Y. Q. Wang, "Acupuncture for pain management: molecular mechanisms of action," The American Journal of Chinese Medicine, vol. 48, no. 4, pp. 793-811, 2020.

[22] F. S. Tonin, I. Rotta, A. M. Mendes, and R. Pontarolo, "Network meta-analysis: a technique to gather evidence from direct and indirect comparisons," Pharmacy Practice, vol. 15, no. 1, p. 943, 2017.

[23] Y.-K. Dai, Y.-B. Wu, R.-L. Li et al., "Efficacy and safety of non-pharmacological interventions for irritable bowel syndrome in adults," World Journal of Gastroenterology, vol. 26, no. 41, pp. 6488-6509, 2020.

[24] Z. Yin, G. Geng, G. Xu, L. Zhao, and F. Liang, "Acupuncture methods for allergic rhinitis: a systematic review and bayesian meta-analysis of randomized controlled trials," Chinese Medicine, vol. 15, no. 1, p. 109, 2020.

[25] T. P. Debray, E. Schuit, O. Efthimiou et al., "An overview of methods for network meta-analysis using individual participant data: when do benefits arise?" Statistical Methods in Medical Research, vol. 27, no. 5, pp. 1351-1364, 2018. 
[26] B. Hutton, G. Salanti, D. M. Caldwell et al., "The PRISMA extension statement for reporting of systematic reviews incorporating network meta-analyses of health care interventions: checklist and explanations," Annals of Internal Medicine, vol. 162, no. 11, pp. 777-784, 2015.

[27] H. MacPherson, D. G. Altman, R. Hammerschlag et al., "Revised STandards for reporting interventions in clinical trials of acupuncture (STRICTA): extending the CONSORT statement," PLoS Medicine, vol. 7, no. 6, Article ID e1000261, 2010.

[28] M. J. Hjermstad, P. M. Fayers, D. F. Haugen et al., "Studies comparing numerical rating scales, verbal rating scales, and visual analogue scales for assessment of pain intensity in adults: a systematic literature review," Journal of Pain and Symptom Management, vol. 41, no. 6, pp. 1073-1093, 2011.

[29] J. A. C. Sterne, J. Savović, M. J. Page et al., "RoB 2: a revised tool for assessing risk of bias in randomised trials," $B M J$, vol. 366, p. 14898, 2019.

[30] J. Mu, A. D. Furlan, W. Y. Lam, M. Y. Hsu, Z. Ning, and L. Lao, "Acupuncture for chronic nonspecific low back pain," Cochrane Database of Systematic Reviews, vol. 12, no. 12, Article ID CD013814, 2020.

[31] A. Cipriani, J. P. T. Higgins, J. R. Geddes, and G. Salanti, "Conceptual and technical challenges in network metaanalysis," Annals of Internal Medicine, vol. 159, no. 2, pp. 130-137, 2013.

[32] A. Sutton, A. E. Ades, N. Cooper, and K. Abrams, "Use of indirect and mixed treatment comparisons for technology assessment," PharmacoEconomics, vol. 26, no. 9, pp. 753-767, 2008.

[33] G. Rücker and G. Schwarzer, "Ranking treatments in frequentist network meta-analysis works without resampling methods," BMC Medical Research Methodology, vol. 15, p. 58, 2015.

[34] E. Baek and J. M. Ferron, "Bayesian analysis for multiplebaseline studies where the variance differs across cases in OpenBUGS," Developmental Neurorehabilitation, vol. 24, no. 2, pp. 130-143, 2021.

[35] G. H. Guyatt, A. D. Oxman, H. J. Schünemann, P. Tugwell, and A. Knottnerus, "GRADE guidelines: a new series of articles in the Journal of Clinical Epidemiology," Journal of Clinical Epidemiology, vol. 64, no. 4, pp. 380-382, 2011.

[36] M. A. Puhan, H. J. Schunemann, M. H. Murad et al., "A GRADE Working Group approach for rating the quality of treatment effect estimates from network meta-analysis," BMJ, vol. 349, p. g5630, 2014.

[37] S. Mehta, R. F. Bastero-Caballero, Y. Sun et al., "Performance of intraclass correlation coefficient (ICC) as a reliability index under various distributions in scale reliability studies," Statistics in Medicine, vol. 37, no. 18, pp. 2734-2752, 2018.

[38] H. Chen and K. He, "Clinical observation of primary trigeminal neuralgia treated by acupuncture," Journal of Practice of Traditional Chinese Medicine, vol. 37, no. 03, pp. 468-469, 2021.

[39] Y. R Chi, "Observation of the effect of acupuncture on primary trigeminal neuralgia," China Health Vision, vol. 142, 2021.

[40] Q. Niu, "Experience in acupuncture treatment of primary trigeminal neuralgia," Women's Health Research, vol. 5, pp. 115-116, 2021.

[41] L. M. Qin, "Clinical observation of 66 cases of primary trigeminal neuralgia treated with acupuncture," Journal of Chinese Clinical medicine, vol. 13, no. 18, pp. 78-79, 2021.
[42] Y. Wang and Q. Zeng, "Effects of acupuncture combined with carbamazepine on quality of life in patients with primary trigeminal neuralgia," Contemporary Medical Symposium, vol. 19, no. 8, pp. 173-174, 2021.

[43] G. R. Wang, "Clinical effect of acupuncture on primary trigeminal neuralgia," World Latest Med Inform, vol. 39, p. 160, 2020.

[44] Y. Q. Ta, Clinical Observation of Acupuncture Combined with Carbamazepine in the Treatment of Primary Trigeminal Neuralgia, Changchun University of Chinese Medicine, Changchun, China, [Chinese], 2020.

[45] Y.-p. Zhang, Y. Wang, W.-g. Xia, and A.-q. Song, "Triple puncture for primary trigeminal neuralgia: a randomized clinical trial," Current Medical Science, vol. 39, no. 4, pp. 638-644, 2019.

[46] W. Hao, Y. Li, and S. Y. Gong, "Clinical observation on treatment of primary trigeminal neuralgia with carbamazepine combined with acupuncture," Journal of Practice of Traditional Chinese Medicine, vol. 35, no. 2, pp. 209-210, 2019, [Chinese].

[47] J. L. Wu, H. Yang, Y. Mi, Y. Song, and Y. Wang, "Clinical study on treatment of primary trigeminal neuralgia by warming acupuncture in xiaguan point," Xinjiang Journal of Traditional Chinese Medicine, vol. 37, no. 3, pp. 34-36, 2019, [Chinese].

[48] J. Xu and H. Wang, "Clinical effect of acupuncture and moxibustion on primary trigeminal neuralgia," Journal Neimenggu of Traditional Chinese Medical Sciences, vol. 38, no. 12, pp. 137-138, 2019, [Chinese].

[49] D. Mu, "Effect analysis of acupuncture on primary trigeminal neuralgia," Chinese Journal of Modern Drug Application, vol. 13, no. 19, pp. 118-120, 2019, [Chinese].

[50] H. Wu, Y. Sun, and Y. Li, "Clinical observation of treating primary trigeminal neuralgia with warm acupuncture and moxibustion in xiaguan acupoint," Journal of Emergency in Traditional Chinese Medicine, vol. 28, no. 08, pp. 1457-1459, 2019, [Chinese].

[51] Y. Liu and C. Liu, "Clinical effect of acupuncture on patients with primary trigeminal neuralgia," Medical Equipment, vol. 32, no. 14, pp. 63-64, 2019, [Chinese].

[52] Y. Wang, "Therapeutic effect of acupuncture on 30 cases of primary trigeminal neuralgia," Yunan Journal Traditional Chinese Medicine, vol. 40, no. 07, pp. 94-54, 2019, [Chinese].

[53] S. Si, "Efficacy of carbamazepine combined with high frequency electroacupuncture in the treatment of primary trigeminal neuralgia," Inner Mongolia Medical Journal, vol. 50, no. 6, pp. 731-733, 2018.

[54] Z. Liang, L. Zhang, and G. Yao, "Randomized parallel controlled study of acupuncture in the treatment of trigeminal neuralgia," Journal Practice of Traditional Chinese Medicine, vol. 12, pp. 67-69, 2018.

[55] S. Huang, "Clinical observation of acupuncture and moxibustion in treating primary trigeminal neuralgia," Chinese Manipulation \& Rehabilitation Medicine, vol. 9, no. 10, pp. 19-20, 2018.

[56] H. Yan, "Clinical observation of acupuncture in treating primary trigeminal neuralgia," Gen J Stomatol, vol. 5, no. 29, pp. 78-82, 2018.

[57] B. Q. Li, Clinical Study on Treatment of Primary Trigeminal Neuralgia with Electroacupuncture, Hubei University of Chinese Medicine, Wuhan, China, [Chinese], 2018.

[58] Z. Y. Huang, Observation on Therapeutic Effect of Local Fire Acupuncture in Treating Primary Trigeminal Neuralgia, 
Hunan University of Chinese Medicine, Changsha, China, [Chinese], 2018.

[59] Y. Li, "Analysis of clinical curative effect of Chinese acupuncture in the treatment of primary trigeminal neuralgia," Chin Foreign Med treatment, vol. 37, no. 10, pp. 177-179, 2018.

[60] Q. M. Long, Clinical Study on the Treatment of Primary Trigeminal Neuralgia (Wind Cold Syndrome) with Acupuncture and Warm Acupuncture, Guangxi University of Chinese Medicine, Guilin, China, [Chinese], 2018.

[61] Y. Ying and H. Zhou, "Carbamazepine combining high frequency electroacupuncture therapy for trigeminal neuralgia and its effects on patients' pain relief and life quality," International Medicine \& Health Guidance News, vol. 24, no. 12, pp. 1806-1808, 2018.

[62] Q. Guo, Z. Wang, H. Yin, and Z. Sun, "Clinical observation of electroacupuncture at jiaji (EX-B2) points for primary trigeminal neuralgia," Shanghai J Acu-mox, vol. 36, no. 4, pp. 392-396, 2017.

[63] Z. Pan, R. Fu, and X. Lin, "Clinical Study on mind-regulating acupuncture in treating primary trigeminal neuralgia," Shanghai J Acu-mox, vol. 36, no. 09, pp. 1067-1073, 2017.

[64] G. H. Su, "Clinical study on treatment of primary trigeminal neuralgia with scalp penetration and electronic acupuncture," Heilongjiang Academy of Chinese Medicine, [Chinese], Harbin, China, 2017.

[65] M. Y. He, Clinical Research of Holographic Acupuncture Combined with Electroacupuncture in the Treatment of Primary Trigeminal Neuralgia, Changchun University of Chinese Medicine, Changchun, China, [Chinese], 2017.

[66] Q. Shen, "40 cases of primary trigeminal neuralgia treated by acupuncture combined with carbamazepine," Traditional Chinese Medical Sciences, vol. 29, no. 09, pp. 63-65, 2016.

[67] F. Xiao and S. Xu, "Clinical study on zhuyu huoluo acupuncture treatment of primary trigeminal neuralgia," Acta Chinese Medicine and Pharmacology, vol. 31, no. 6, pp. 918-921, 2016.

[68] S. Feng, "Efficacy of carbamazepine combined with acupuncture in the treatment of primary trigeminal neuralgia," Journal of Clinical Medicine, vol. 3, no. 9, pp. 1714-1715, 2016.

[69] K. Li, "Clinical value of acupuncture and moxibustion in treating primary trigeminal neuralgia," J Neimenggu of Traditional Chinese Medicine, vol. 35, no. 11, p. 120, 2016.

[70] F. Zhang, "Clinical observation of primary trigeminal neuralgia treated by TCM acupuncture," World Latest Med Inform, vol. 16, no. 63, pp. 181-182, 2016.

[71] Q. Wang, Clinical Observation of Primary Trigeminal Neuralgia by Electroacupuncture Bangci Xiaguan Point Treatment, Heilongjiang University of Chinese Medicine, Harbin, China, [Chinese], 2016.

[72] L. Zhou and Y. Ying, "Clinical study of acupuncture in treating 65 cases of primary trigeminal neuralgia," China Medical Device Inform, vol. 22, no. 10, pp. 107-108, 2016.

[73] Y. Liu, "Clinical curative observation of using acupuncture and moxibustion in the treatment of primary trigeminal Neuralgia," J Sichuan of Traditional Chinese Medicine, vol. 34, no. 04, pp. 183-185, 2016.

[74] H. Xie, "Clinical observation of acupuncture in treating primary trigeminal neuralgia," World Latest Med Inform, vol. 16, no. 05, 2016.
[75] K. Liu, "Trigeminal neuralgia parallel randomized controlled study of acupuncture treatment," J Practical Traditional Chinese Medicine, vol. 06, pp. 139-140, 2015.

[76] H. Wang, "An effective analysis of acupuncture on 70 cases of trigeminal neuralgia," Clin J Chin Med, vol. 7, no. 23, pp. 44-45, 2015.

[77] W. Xia, "Clinical observation of acupuncture in treating primary trigeminal neuralgia," Guid Chin Med, vol. 13, no. 14, pp. 202-203, 2015.

[78] J. Wang and J. Liu, "Clinical observation of acupuncture and moxibustion in treating primary trigeminal neuralgia," J Neimenggu of Traditional Chinese Medicine, vol. 33, no. 36, p. 47, 2014.

[79] Z. Zhou, W. Wang, J. Liu, and S. Gu, "Clinical observation of 30 cases of primary trigeminal neuralgia treated by electroacupuncture through acupoint," Chin J Trad Med Sci Tech, vol. 21, no. 06, p. 643, 2014.

[80] S. T. Liu, Observation on Therapeutic Effect of Acupuncture for Treatment of Trigeminal Neuralgia Yangming Meridian, Hunan University of Chinese Medicine, Changsha, China, [Chinese], 2014.

[81] D. Xie, "Effect of different acupuncture methods on primary trigeminal neuralgia," Chin Foreign Med Treatment, vol. 33, no. 12, pp. 136-137, 2014.

[82] Y. Li, "Clinical observation on the treatment of primary trigeminal neuralgia by integrated traditional Chinese and western medicine," Chin Health Ind, vol. 11, no. 5, pp. 189-190, 2014.

[83] C. Zhang, "Analysison Curative Effect of Electric head acupuncture therapy of primary trigeminal neuralgia," Liaoning J Traditional Chinese Medicine, vol. 40, no. 6, pp. 1214-1215, 2013.

[84] L. Wang, L. Huang, Q. Luo, Y. Wang, and K. Chen, “Clinical efficacy observation on primary trigeminal neuralgia with acupuncture," J Clin Acu-Mox, vol. 29, no. 07, pp. 28-30, 2013.

[85] C. J. Luo, The Clinical Research on Treatment of Classic Trigeminal Neuralgia by the Method of Ataralgesia Acupuncture, Heilongjiang University of Chinese Medicine, Harbin, China, [Chinese], 2013.

[86] P. Zhao and H. Zhou, "60 cases of primary trigeminal neuralgia with blood stasis zheng treated by acupuncture," Journal of Traditional Chinese Medical Sciences, vol. 03, no. 10, p. 130, 2011.

[87] J. Zheng and J. Shi, "Therapeutic effect of electro-acupuncture on primary trigeminal neuralgia observation," J Neimenggu of Traditional Chinese Medicine, vol. 30, no. 1, pp. 62-64, 2011.

[88] Q. Han, "Clinical observation of electroacupuncture in treating 30 cases of primary trigeminal neuralgia," Guid J Traditional Chinese Medicine Pharma, vol. 15, no. 9, p. 35, 2009.

[89] N. Zhao and C. Jia, "Clinical observation of abdominal acupuncture in the treatment of primary trigeminal neuralgia," Mod Traditional Chinese Medicine, vol. 29, no. 6, pp. 47-48, 2009.

[90] R. Li, "Observation on the curative effect of acupuncture and moxibustion in treating primary trigeminal neuralgia," Chin Foreign Med treatment, vol. 28, no. 20, p. 116, 2009.

[91] Y. Jiao, J. Li, H. Luo, D. Ding, D. Wei, and J. Zhang, "Clinical study of acupuncture in treating trigeminal neuralgia," Journal of Emergency in Traditional Chinese Medicine, vol. 17, no. 3, pp. 323-325, 2008.

[92] Y. P. Zhang, Clinical Study of Qici of Acupuncture on Primary Trigeminal Neuralgia, Hubei University of Chinese Medicine, Wuhan, China, [Chinese], 2006. 
[93] Z. Zhou, J. Li, and H. Luo, "Observation on therapeutic effect of triple needling on primary trigeminal neuralgia," Zhongguo Zhen Jiu, vol. 24, no. 12, pp. 835-836, 2004.

[94] V. Tentolouris-Piperas, G. Lee, J. Reading, A. G. O’Keeffe, J. M. Zakrzewska, and R. Cregg, "Adverse effects of antiepileptics in trigeminal neuralgiform pain," Acta Neurologica Scandinavica, vol. 137, no. 6, pp. 566-574, 2018.

[95] H. Naci, G. van Valkenhoef, J. P. T. Higgins, R. Fleurence, and A. E. Ades, "Evidence-based prescribing: combining network meta-analysis with multicriteria decision analysis to choose among multiple drugs," Circulation: Cardiovascular Quality and Outcomes, vol. 7, no. 5, pp. 787-792, 2014.

[96] D. M. Caldwell, A. E. Ades, and J. P. T. Higgins, "Simultaneous comparison of multiple treatments: combining direct and indirect evidence," $B M J$, vol. 331, no. 7521, pp. 897-900, 2005.

[97] G. Salanti, "Indirect and mixed-treatment comparison, network, or multiple-treatments meta-analysis: many names, many benefits, many concerns for the next generation evidence synthesis tool," Research Synthesis Methods, vol. 3, no. 2, pp. 80-97, 2012.

[98] M. Cumpston, T. Li, M. J. Page et al., "Updated guidance for trusted systematic reviews: a new edition of the Cochrane Handbook for Systematic Reviews of Interventions," Cochrane Database of Systematic Reviews, vol. 10, Article ID ED000142, 2019.

[99] L. Turner, L. Shamseer, D. G. Altman et al., "Consolidated standards of reporting trials (CONSORT) and the completeness of reporting of randomised controlled trials (RCTs) published in medical journals," Cochrane Database of Systematic Reviews, vol. 11, no. 11, Article ID MR000030, 2012.

[100] X. Li, Q. Dai, Z. Shi et al., "Clinical efficacy and safety of electroacupuncture in migraine treatment: a systematic review and network meta-analysis," The American Journal of Chinese Medicine, vol. 47, no. 8, pp. 1755-1780, 2019. 